\title{
SOCIAL INSTABLITY
}

1 Social Instability is an Effective Chronic Stress Paradigm for both Male and Female Mice

2

14 *Address correspondence to:

15 Benjamin Adam Samuels, PhD

16 Assistant Professor

17 Behavioral \& Systems Neuroscience

18 Department of Psychology

19 Busch Campus Psychology Building Room 215

20 Rutgers University - New Brunswick

21152 Frelinghuysen Road, Piscataway, NJ 08854

22 Phone: +1-848-445-8933

23 Email: ben.samuels@rutgers.edu 


\section{SOCIAL INSTABLITY}

\section{ABSTRACT:}

Despite stress-associated disorders having a higher incidence rate in females, preclinical

29 research mainly focuses on males. Chronic stress paradigms, such as chronic social defeat and

30 chronic corticosterone administration, were mainly designed and validated in males and

31 subsequent attempts to use these paradigms in females has demonstrated sex differences in the

32 behavioral and HPA axis response to stress. Here, we developed a social stress paradigm, social

33 instability stress (SIS), which exposes adult mice to unstable social hierarchies for 7 weeks. SIS

34 effectively induces negative valence behaviors and hypothalamus-pituitary-adrenal (HPA) axis

35 activation in both males and females. Importantly, while there were effects of estrous cycle on

36 behavior, this variability did not impact the overall effects of SIS on behavior, suggesting estrous

37 does not need to be tracked while utilizing SIS. Furthermore, the effects of SIS on negative

38 valence behaviors were also reversed following chronic antidepressant treatment with fluoxetine

39 (FLX) in both males and females. SIS also reduced adult hippocampal neurogenesis in female

40 mice, while chronic FLX treatment increased adult hippocampal neurogenesis in both males and

41 females. Overall, these data demonstrate that the SIS paradigm is an ethologically valid approach

42 that effectively induces chronic stress in both adult male and adult female mice. 


\section{SOCIAL INSTABLITY}

\section{INTRODUCTION}

44 Several mood disorders, including anxiety and depression, are commonly thought to be

45 precipitated and/or exacerbated by chronic exposure to stressful experiences. These stress-

46 associated mood disorders occur at higher rates in women than in men. However, historically

47 preclinical stress studies are notorious for only including males (1) and most chronic stress

48 experimental paradigms are validated only using male rodents (2-4). One possible reason is that

49 the female estrous cycle has well-documented effects on neural functions and behavior (5-8).

50 However, it is unlikely that the estrous cycle contributes significantly more variability to female

51 than male rodents $(9,10)$. This realization, coupled with a mandate from the United States

52 National Institutes of Health (NIH) to include both sexes in grant applications, has led to an

53 increase in behavioral neuroscience studies that include both sexes (11). However, while some

54 progress has been made, preclinical studies incorporating chronic stress still lag behind because

55 many of the experimental paradigms were originally designed and optimized only for male

56 rodents $(1,12-15)$.

57 One commonly used chronic stress paradigm in preclinical studies, unpredictable chronic

58 mild stress (UCMS), differentially affects negative valence behaviors and HPA axis activation in

59 male and female mice (16-19). Furthermore, UCMS has historically been plagued with

60 reproducibility and validity issues (20-22). Another paradigm widely used in preclinical studies

61 is chronic social defeat stress (CSDS), where rodents are subjected to larger more aggressive

62 conspecifics (14). In males, CSDS results in activation of the hypothalamic-pituitary-adrenal

63 (HPA) axis and increased negative valence behaviors $(23,24)$. However, standard CSDS

64 protocols are not effective for stressing female rodents (25) unless variations that may infringe 


\section{SOCIAL INSTABLITY}

65 upon ethological validity such as activating the ventral medial hypothalamus of aggressors or

66 applying male urine to females are made $(26,27)$.

67 In both males and females the HPA axis is activated by stress $(28,29)$, resulting in the

68 release of the stress hormone cortisol (human) or corticosterone (rodents), and HPA axis

69 dysregulation is found in many patients with mood disorders (30). Therefore, a distinct paradigm

70 that effectively mimics chronic stress in male rodents is chronic administration of corticosterone

71 (CORT). However, similar to CSDS, chronic CORT was developed in male rodents $(13,31,32)$.

72 In females, postpartum chronic administration of CORT increases immobility time within the

73 forced swim test (FST) and decreases maternal behavior in female rats $(33,34)$. However, one

74 recent study also suggests that chronic CORT is not as effective in producing increases in

75 negative valence behaviors in females as in males (35).

76 Thus, there is currently no widely used chronic stress paradigm that is effective in both

77 adult male and adult female rodents and many preclinical labs are either utilizing distinct

78 paradigms for males and females or continuing to focus exclusively on males. Development of a

79 single chronic stress paradigm that is effective in both sexes allowing direct comparisons and is

80 ethologically valid is necessary for advancing our understanding of how chronic stress impacts

81 neural function and behavior. Here, we describe the development of a social instability stress

82 (SIS) paradigm that is effective in both adult male and adult female mice. The social instability

83 paradigm described here involves exposure to unstable social hierarchical dynamics for several

84 weeks and effectively induces negative valence behaviors, HPA axis activation, and neural

85 changes associated with chronic stress such as altered adult hippocampal neurogenesis in both

86 adult males and adult females of the widely used C57BL/6J strain. Furthermore, the negative

87 valence behaviors induced by social instability in both adult males and adult females can be 


\section{SOCIAL INSTABLITY}

88 reversed by subsequent administration with the antidepressant fluoxetine, lending

89 pharmacological validity to this chronic stress paradigm. Taken together, our results support the

90 usage of social instability as an ethologically valid chronic stress paradigm that is effective for

91 both adult male and adult female rodents.

92 


\section{SOCIAL INSTABLITY}

\section{MATERIALS AND METHODS}

\section{Mice}

95 Adult 8 week old female and male C57BL/6J mice were purchased from Jackson laboratories

96 and maintained on a 12L:12D schedule with ad libitum food and water. All testing was

97 conducted in compliance with the NIH laboratory animal care guidelines and approved by

98 Rutgers University Institutional Animal Care and Use Committee.

\section{Chronic Stress Paradigms}

101 Chronic Corticosterone. Adult male and female C57BL/6J mice were randomly assigned to

102 either vehicle (VEH) or corticosterone (CORT) treatment, with weights measured once per week

103 during treatments. Corticosterone (35 ug/mL, equivalent to $5 \mathrm{mg} / \mathrm{kg} /$ day) was dissolved in $0.45 \%$

104 beta-cyclodextrin (Sigma) water and delivered ad libitum in opaque drinking bottles (David et

105 al., 2009). VEH mice received $0.45 \%$ beta-cyclodextrin water ad libitum. Following 4 weeks of

106 CORT or VEH treatment, mice received 3 weeks of fluoxetine (FLX) (18 mg/kg/day) or VEH

107 (water) via oral gavage with CORT or VEH remaining in the drinking water throughout

108 antidepressant treatment (timeline in Figure 1A). On behavioral testing days FLX or VEH was

109 administrated after mice completed the behavioral tests to avoid acute effects. The following 4

110 groups emerged: VEH+VEH, VEH+FLX, CORT+VEH, and CORT+FLX.

112 Social Instability Stress (SIS). Adult male and female C57BL/6J mice were randomly assigned to

113 either SIS or control (CNTRL, no stress). SIS mice experienced unstable social hierarchies,

114 where social dynamics were changed twice a week for 7 weeks (adapted from 36-38). During the

1157 weeks, a cage composition change would consist of an individual mouse being introduced to 3- 


\section{SOCIAL INSTABLITY}

1164 novel mice of the same sex, with total mice per cage ranging from 3-5 mice. The rotation

117 schedule was randomized to prevent mice from having an encounter with a recent cage mate. At

118 the end of the 7 weeks, SIS mice remained housed with the mice of the last cage composition

119 (adapted from 36). Male and female CNTRL mice had the same cage mates throughout the entire

120 paradigm and were subjected to cage changes twice per week (adapted from 36, 39). Subsequent

121 to SIS exposure, SIS and CNTRL mice were randomly assigned to receive either FLX (18

$122 \mathrm{mg} / \mathrm{kg} /$ day) or $\mathrm{VEH}$ (water) via oral gavage (timeline in Figure 2A). The following 4 groups

123 emerged: CNTRL+VEH, CNTRL+FLX, SIS+VEH, sand SIS+FLX.

\section{Vaginal Lavage}

126 Vaginal lavages were performed daily during the stress paradigms and two weeks prior to

127 behavioral testing to ensure mice were cycling throughout all four stages of the estrous cycle

128 regularly. After completing each behavioral test, vaginal smears were collected to assess the

129 estrous state mice were in during the behavior test. Samples were collected via a pipette filled

130 with $\mathrm{ddH}_{2} \mathrm{O}$ gently expelled and placed at the vaginal canal opening (without penetration).

131 Samples were suctioned back into the pipette, placed on a microscope slide, and dried on a slide

132 warmer before imaged with an EVOS FL Auto 2.0 microscope (Thermofisher Scientific) at 10x

133 magnification $(40,41)$. Estrous phases were identified by the presence or absence of nucleated

134 epithelial cells, cornified epithelial cells, and leukocytes (41, 42 Figure 3B).

\section{Behavioral Testing}

137 Open Field $(O F)$. Motor activity was quantified via infrared photobeams in Plexiglass open field

138 boxes $43 \times 43 \mathrm{~cm}^{2}$ (Kinder Scientific). As previously described (13), activity chambers were 


\section{SOCIAL INSTABLITY}

139 computer interfaced for data sampling at 100ms resolution. The computer software predefines

140 grid lines that divide each OF chamber into center and periphery regions, with the center being a

141 square $11 \mathrm{~cm}$ from the wall. For our analyses we calculated percent distance traveled in the center

142 ((center distance/total distance traveled $) * 100)$.

144 Light Dark Test (LDT). LDT was conducted in OF chambers with, a dark plastic box (opaque to

145 visible light, but transparent to infrared light) covering $1 / 3$ of the arena, inserted to separate the

146 OF into light and dark compartments. The dark box contained an opening that allowed passage

147 between the light and dark (13), with the light compartment brightly illuminated (1000 lux;

148 Kinder Scientific). At the beginning of each 5-minute test, mice were placed in the dark

149 compartment, with distance traveled in the light ((distance traveled in the light/total distance)

$150 * 100)$ used for analyses.

152 Elevated Plus Maze (EPM). The EPM test consisted of a plus-shaped apparatus with two open

153 and two closed arms (side walls), elevated 2 feet from the floor. During the five-minute test, the

154 mice were recorded from a video camera mounted on the ceiling above each EPM arena.

155 EthoVision (Noldus) software was used to quantify the data, with distance traveled in open arms

156 ((total open arm distance/total distance traveled)*100) used for analyses.

158 Forced Swim Test (FST). A modified FST procedure suitable for mice was used (13), with

159 individual cylinders $(46 \times 32 \times 30 \mathrm{~cm})$ filled with room-temperature water $\left(25-26^{\circ} \mathrm{C}\right)$. Two sets of

160 photobeams were mounted on opposite sides of the cylinder (Kinder Scientific) to record 


\section{SOCIAL INSTABLITY}

161 swimming behavior during the 6-minute test. Immobility was assessed during only the last 4-

162 minutes of the test since mice habituate to the task during the initial 2-minutes.

164 Novelty Suppressed Feeding (NSF). Mice were food deprived for 18 hours within their home

165 cage, prior to being placed in the corner of a testing apparatus $(50 \times 50 \times 20 \mathrm{~cm})$ filled with $2 \mathrm{~cm}$ of

166 corncob bedding, with a single food pellet attached to a white platform in the brightly

167 illuminated center (1500 lux). The NSF test lasted 6 minutes with latency to eat (defined as the

168 mouse sitting on its haunches and biting the pellet with the use of forepaws) recorded in seconds.

169 Mice that timed out were assigned a latency of 360 secs. Immediately after the test, mice were

170 transferred to their home cages and given ad libitum access to food for 5 minutes. Latency to eat

171 and amount of food consumed in home cage was measured as a control for feeding behavior

172 observed in the NSF.

173

174 Brain Collection, Sectioning, and Immunohistochemistry

175 Brain Collection and Sectioning. Following behavioral testing, brains were collected from all

176 experimental mice. Mice were anesthetized with ketamine $(80 \mathrm{mg} / \mathrm{kg})$ and perfused transcardially

177 with PBS followed by 4\% paraformaldehyde. Brains were collected, stored in 4\%

178 paraformaldehyde overnight at $4^{\circ} \mathrm{C}$, and then switched to $30 \%$ sucrose $0.1 \%$ sodium azide

$179\left(\mathrm{NaN}_{3}\right)$ in PBS solution until sectioned. Using a cryostat, the hippocampus was collected (43;

180 Bregma -1.22 to -3.88) and mounted on SuperfrostPlus slides (Thermofisher Scientific) and

181 stored at $-20^{\circ} \mathrm{C}$ until immunostaining. 


\section{SOCIAL INSTABLITY}

183 Immunohistochemistry. The effects of SIS and FLX on adult hippocampal neurogenesis were

184 assessed across the entire hippocampus by counting 1 out of every 6 hippocampal sections.

185 Slides were washed in 1\% TritonX-100-PBS and then 3 PBS washes. Next, slides were

186 incubated in citrate buffer for 30 minutes followed by 3 PBS washes. Slides were blocked for 1

187 hour in $10 \%$ normal goat serum (NGS) diluted in PBS before being incubated overnight at $4^{\circ} \mathrm{C}$

188 in either anti-rabbit Ki67 (1:500; Abcam, ab16667) or doublecortin anti-rabbit (1:500; Life

189 technologies; 481200) diluted in 2\%NGS-PBS. Following 18 hours of incubation, 3 PBS washes

190 were performed and then 2 hours of incubation in CY-5 goat anti-rabbit (1:1000, Invitrogen,

191 ThermoFisher Scientific, A10523) diluted in 2\%NGS-PBS. Following 3 more PBS washes,

192 slides were counterstained with DAPI (1:15000; ThermoFisher Scientific) for 15 minutes, with a

193 final PBS wash before cover slipping using prolong diamond (ThermoFisher Scientific). High-

194 resolution fluorescent images were taken using an EVOS FL Auto 2.0 microscope (Thermofisher

195 Scientific) at 10x magnification for quantification of $\mathrm{Ki}^{+}$(Figure 5A) or $\mathrm{DCX}^{+}$cells (Figure

196 5C) and counted across a total of 12 sections of hippocampus. $\mathrm{Ki}^{+} 7^{+}$cells were overlaid with

197 DAPI for quantification purposes. Images were also taken at 40x magnification to subcategorize

$198 \mathrm{DCX}^{+}$cells according to their dendritic morphology: $\mathrm{DCX}^{+}$cells with no tertiary dendritic

199 processes and $\mathrm{DCX}^{+}$cells with complex, tertiary dendrites (Figure 5E). The maturation index

200 was defined as the ratio of $\mathrm{DCX}^{+}$cells possessing tertiary dendrites over the total $\mathrm{DCX}^{+}$cells.

\section{Blood Collection and Corticosterone ELISA}

203 Mice were weighed to ensure that non-terminal blood collection was no more than $1 \%$ of the

204 mouse's body weight. Prior to SIS, all mice had baseline blood samples collected from the left

205 retro-orbital sinus in accordance with IACUC guidelines. To measure corticosterone levels in 


\section{SOCIAL INSTABLITY}

206

207

208

209

210

211

212

213

214

215 Total yield of plasma per blood collection was between 25 to $40 \mu \mathrm{L}$. To assess differences in

216 plasma corticosterone in response to SIS stress and EPM behavior, blood was analyzed from

217 each time point (prior to SIS, SIS cage change, EPM) across 5 mice per sex per stress condition

218 (male: $\mathrm{CNTRL}=5$, SIS=5; female: $\mathrm{CNTRL}=5, \mathrm{SIS}=5$ ). Each sample (total of $\mathrm{n}=60$ across all time

219 points) was diluted 1:100 and assayed in triplicate according to the manufacturer's protocol

220 (Arbor Assays Corticosterone ELISA Kit).

\section{Statistical Analyses}

223 All analyses were conducted using Graph Pad Prism 7. Sex differences for both CORT and SIS

224 paradigms were analyzed using 2x4 analysis of variance (ANOVA) with follow-up Bonferroni

225 post-hoc comparisons. Further analyses in the SIS paradigm used 2x2 ANOVAs within sex to

226 compare stress (SISvsVEH) and FLX (FLXvsVEH) effects. Given that the NSF data fails to

227 meet basic assumptions of normality, Kaplan Meier survival analysis (nonparametric test) was

228 used to analyze sex differences in feeding behavior. Lastly, to analyze differences in exogeneous 


\section{SOCIAL INSTABLITY}

229 corticosterone levels across the three time points (prior to SIS, SIS cage change, and EPM

230 exposure), time point, sex, and groups were used to conduct a $3 \times 2 \times 2$ repeated measures

231 ANOVA. These analyses of corticosterone levels were then followed up with separate $2 \times 3$

232 repeated measures ANOVAs within each sex. 
SOCIAL INSTABLITY

\section{RESULTS}

\section{Behavioral Sex Differences following Chronic CORT Administration}

236 Chronic CORT administration is used to mimic chronic stress in male rodents $(13,31)$.

237 However, there are inconsistencies in published reports about whether CORT administration is

238 also effective in female rodents (33-35). Therefore, we chronically administered exogenous

239 CORT $(5 \mathrm{mg} / \mathrm{kg} /$ day) or VEH to male and female C57BL/6J mice (timeline in Figure $1 \mathrm{~A})$.

240 Following 4 weeks of CORT, mice received either FLX $(18 \mathrm{mg} / \mathrm{kg})$ or VEH, resulting in four

241 groups: VEH+VEH (male=10; female=10), VEH+FLX (male=10; female=10), CORT+VEH

$242($ male=10; female $=10)$, and CORT+FLX (male=10; female=9). We examined CORT and FLX

243 effects across these groups in negative valence behavioral measures known to be affected by

244 chronic stress paradigms: open field (OF), light/dark test (LDT), elevated plus maze (EPM),

245 novelty suppressed feeding (NSF), and forced swim test (FST) respectively.

246 First, separate $2 \times 4$ ANOVAs ( $\operatorname{sex} x$ group) were used to investigate potential sex

247 differences. Significant sex effects were found in LDT light distance traveled $\left(F_{(1,72)}=13.69\right.$,

$248 \quad p<0.001$; Figure 1C small panel $)$ and EPM open arms $\left(F_{(1,72)}=9.97, p<0.001\right.$; Figure 1D small

249 panel), with CORT+VEH males traveling less distance in the light $(p=0.001)$ and in EPM open

$250 \operatorname{arms}(p=0.005)$ than CORT+VEH females (Figure 1C-D small panels). Additionally, sex

251 differences in NSF latency to eat were discovered by Kaplan Meier survival analyses (log-rank

252 Mantel Cox test), with CORT+VEH males having a longer latency to eat than CORT+VEH

253 females $\left(\chi^{2}(1)=37.35, p<0.001\right.$; Figure $\left.1 \mathrm{G}\right)$. No sex differences were observed between groups

254 in the $\mathrm{OF}(p=0.97)$ and FST $(p=0.17)$. Taken together, these data demonstrate that CORT

255 administration more effectively induces negative valence behaviors in males than in females. 
SOCIAL INSTABLITY

After examining sex differences, separate $2 \times 2$ ANOVAs were run to investigate CORT

257 and FLX effects on negative valence behaviors within each sex. Within males significant

$258 \operatorname{CORT}\left(F_{(1,36)}=13.53, p=0.0008\right)$ and $\operatorname{FLX}\left(F_{(1,36)}=9.88, p=0.0033\right)$ effects on LDT light

259 distance traveled (Figure 1C) were found, with CORT+VEH males traveling less than

$260 \mathrm{VEH}+\mathrm{VEH}(p=0.0007)$ and CORT $+\mathrm{FLX}(p=0.0017)$ males (Figure 1C larger panel; Bonferroni

261 corrected). In the EPM, significant FLX $\left(F_{(1,36)}=25.68, p<0.0001\right)$ and interaction $\left(F_{(1,36)}=5.29\right.$,

$262 p=0.027)$ effects were observed in open arm distance traveled, with CORT+VEH males

263 traveling less in the open arms than VEH+VEH $(p=0.025)$ and CORT $+\mathrm{FLX}(p=0.0042)$ males

264 (Figure 1D larger panel; Bonferroni corrected). Additionally, in males a significant effect of

$265 \operatorname{FLX}\left(F_{(1,36)}=39.38, p<0.00001\right)$ was observed on FST immobility time, with CORT + FLX

266 males being less immobile than CORT+VEH males $(p<0.001$, Bonferroni corrected; Figure

267 1E). VEH+FLX males were also less immobile than VEH+VEH males $(p=0.0007$; Bonferroni

268 corrected; Figure 1E). Lastly, Kaplan Meier survival analysis revealed a significant difference

269 between male groups $\left(\chi_{(3)}^{2}=37, p<0.0001\right)$, with further analyses revealing CORT + VEH males

270 had a longer latency to eat than $\mathrm{VEH}+\mathrm{VEH}\left(\chi_{(1)}^{2}=37.58, p<0.0001\right.$; Bonferroni corrected $)$ and

$271 \operatorname{CORT}+\mathrm{FLX}\left(\chi_{(1)}^{2}=15.03, p<0.0001\right.$; Bonferroni corrected; Figure 1F-H).

272 In females, separate 2x2 ANOVAs investigating the effects of CORT and FLX showed a

273 significant FLX effect in the $\operatorname{EPM}\left(F_{(1,36)}=5.88, p=0.02\right)$ and $\operatorname{FST}\left(F_{(1,35)}=28.37, p<0.0001\right)$,

274 with VEH+FLX females spending more time in the open arms $(p=0.018$, Bonferroni corrected;

275 Figure 1D) and less time immobile $(p<0.0001$; Figure 1E) than VEH+VEH females.

276 CORT+FLX females also spent less time immobile in the FST than CORT+VEH females

$277(p=0.018$; Figure 1E). Lastly, Kaplan Meier survival analysis revealed a significant difference

278 between female groups $\left(\chi^{2}{ }_{(3)}=9.28, p=0.026\right)$, with further analyses revealing VEH+FLX 


\section{SOCIAL INSTABLITY}

279 females had a shorter latency to eat than VEH+VEH females $\left(\chi_{(1)}^{2}=4.54, p=0.033\right.$; Bonferroni

280 corrected; Figure 1F). Taken together, these data further demonstrate that while FLX has

281 behavioral effects in both males and females, CORT administration only mimics the effects of

282 chronic stress in males. These data support the findings of Mekiri and colleagues (35) and

283 suggest that CORT administration is not a useful paradigm for studying stress effects in

284 females.

\section{SIS is Effective in both Males and Females}

Since chronic CORT appeared to only mimic the behavioral effects of chronic stress in

288 males, we next developed a social instability paradigm that we reasoned would be stressful for

289 both sexes. To this end, C57BL/6J mice were exposed to unstable same sex hierarchical

290 conditions within their housing environment (Figure 2A). Specifically, SIS mice experience a

291 change in cage dynamics every 3 days for 7 weeks, while CNTRL are housed with the same

292 cage mates for the duration of the experiment. Adult male and adult female C57BL/6J mice

293 were assigned to either SIS or CNTRL (no stress) housing conditions for 7 weeks, and then

294 received 3 weeks of either VEH or FLX $(18 \mathrm{mg} / \mathrm{kg}$ daily) resulting in the following groups:

295 CNTRL+VEH (male=20; female=20), CNTRL+FLX (male=10; female=10), SIS+VEH

296 (male=25; female=28); SIS+FLX (male=33; female=35). To analyze whether sex differences

297 existed within the four groups (CNTRL+VEH, CNTRL+FLX, SIS+VEH, and SIS+FLX), 2x4

298 ANOVAs (sex x group) were run for each behavior. All 2x4 ANOVAs analyses revealed no

299 main effect of sex for any measure: distance traveled in OF center $(p=0.11)$, LDT light distance

$300(p=0.44)$, EPM open arm distance $(p=0.65)$, FST immobility $(p=0.17)$ (Figures 2B-F small

301 panels). For NSF, Kaplan Meier survival curves also showed no sex differences across the four 


\section{SOCIAL INSTABLITY}

302 groups $(p=0.33)$ (Figure $2 \mathrm{~F}-\mathrm{H})$. These data suggest that SIS may be impacting males and

303 females similarly. Thus, to determine the effectiveness of SIS exposure and subsequent FLX

304 treatment in both males and females, 2x2 ANOVAs (SISxFLX) were run within each sex.

305 In males, a 2x2 ANOVA (SISxFLX) revealed significant SIS effect $\left(F_{(1,83)}=4.148\right.$,

$306 p=0.0449)$ and interaction $\left(F_{(1,83)}=4.087, p=0.0464\right)$ in OF center distance traveled, with

307 SIS +VEH males traveling less distance than CNTRL+VEH males $(p=0.0041$; Figure $2 \mathrm{~B}$ larger

308 panel; Bonferroni corrected). Significant main effects of FLX $\left(F_{(1,83)}=10.53, p=0.0017\right)$ and an

309 interaction $\left(F_{(1,83)}=7.076, p=0.0094\right)$ were found in LDT light traveled, with SIS+VEH males

310 traveling less in the light than CNTRL+VEH males $(p=0.029)$ and SIS + FLX $(p<0.0001$; Figure

$3112 \mathrm{C}$ larger panel; Bonferroni corrected). In the EPM, there was a significant effect of FLX

$312\left(F_{(1,83)}=13.25, p=0.0005\right)$, with CNTRL+VEH males traveling less in EPM open arms than

313 CNTRL+FLX ( $p=0.0161$; Bonferroni corrected $)$ and SIS+VEH males traveling less than

314 SIS+FLX males ( $p=0.0307$; Figure 2D larger panel; Bonferroni corrected). Additionally,

315 significant $\operatorname{SIS}\left(F_{(1,46)}=7.1, p=0.0106\right)$ and $\operatorname{FLX}\left(F_{(1,46)}=18.92, p<0.0001\right)$ effects were

316 observed in FST immobility time. Specifically, CNTRL+VEH males were more immobile than

317 CNTRL+FLX males $(p=0.0144)$, and SIS+VEH males were more immobile than SIS+FLX

318 males ( $p=0.0026$; Figure 2E large panel; Bonferroni corrected). Lastly, in the NSF, there was a

319 significant difference in Kaplan Meier survival curves of latency to eat across groups $\left(\chi^{2}{ }_{(3)}=\right.$

$32034.06, p<0.0001)$, with SIS+VEH males having a longer latency to feed than CNTRL+VEH

$321 \quad\left(\chi^{2}{ }_{(1)}=33.7, p<0.0001\right.$; Bonferroni corrected $)$ and $\operatorname{SIS}+\mathrm{FLX}\left(\chi^{2}(1)=25.1, p<0.0001\right.$; Bonferroni

322 corrected) males (Figure 2F, 2G). Overall in males SIS had a significant effect on OF, LDT,

323 FST, and NSF behaviors, with FLX treatment reversing the effects of SIS stress in LDT, EPM,

324 FST, and NSF. 
SOCIAL INSTABLITY 5.017, $p=0.0276)$ and an interaction $\left(F_{(1,91)}=4.643, p=0.0339\right)$ effect in the OF, with SIS+VEH

327 females traveling less in the center than CNTRL+VEH females $(p=0.0019$; Figure 2B larger

328 panel; Bonferroni corrected). In the LDT, a significant FLX $\left(F_{(1,91)}=14.47, p=0.0003\right)$ effect

329 was observed, with SIS+VEH females traveling less in the light than SIS+FLX $(p<0.0001)$ and

$330 \mathrm{CNTRL}+\mathrm{VEH}(p=0.0471$; Figure $2 \mathrm{C}$; Bonferroni corrected) females. Within the EPM, a

331 significant FLX $\left(F_{(1,91)}=22.86, p<0.0001\right)$ effect was observed, with SIS+VEH females

332 traveling less distance in the open arms than SIS+FLX females $(p=0.0004)$ and CNTRL+VEH

333 traveling less than CNTRL+FLX females ( $p=0.0039$; Figure 2D; Bonferroni corrected).

334 Furthermore, significant main effects of SIS $\left(F_{(1,46)}=6.4, p=0.014\right)$ and FLX $\left(F_{(1,46)}=24.92\right.$,

$335 p<0.0001)$ were observed in FST immobility time, with SIS+VEH females more immobile than

336 SIS+FLX females $(p=0.0006)$ and CNTRL+VEH females more immobile than CNTRL+FLX

337 females ( $p=0.0043$; Figure 2F; Bonferroni corrected). Lastly, in the NSF, there was a

338 significant difference in Kaplan Meier survival curves of latency to eat across groups $\left(\chi^{2}(3)=\right.$

$33916.41, p=0.0009)$, with SIS+VEH females having a longer NSF latency to feed than

$340 \mathrm{CNTRL}+\mathrm{VEH}\left(\chi_{(1)}^{2}=29.5, p<0.0001\right.$; Bonferroni corrected $)$ and $\operatorname{SIS}+\mathrm{FLX}\left(\chi_{(1)}^{2}=4.09\right.$,

$341 \quad p=0.040$; Figure 2F, 2H, Bonferroni corrected) females. In females, as seen in males, it

342 appeared that SIS had an impact on OF, FST, and NSF behaviors, with FLX treatment

343 reversing the effects of SIS stress in LDT, EPM, FST, and NSF. Taken together, these data

344 suggest that SIS effectively induces negative valence behaviors in both adult male and adult

345 female mice. 


\section{SOCIAL INSTABLITY}

Since SIS impacted female behavior, we next examined the 4 stages of the estrous cycle

349 (Proestrus, Estrus, Metestrus, Diestrus) in CNTRL and SIS females following each behavioral

350 assay (Figure 3A). For this experiment FLX was not used. To determine SIS and estrous cycle

351 effects on behavior, separate 2x4 ANOVAs (SIS $x$ Estrous) were run for each behavioral assay.

352 In the OF, significant estrous cycle $\left(F_{(3,39)}=45.16, p<0.001\right)$ and $\operatorname{SIS}\left(F_{(1,39)}=19.13, p<0.0001\right)$

353 effects were observed. Specifically, SIS estrus females traveled less in the center than proestrus

$354(p=0.0003)$ and metestrus $(p=0.013)$ females. Additionally, SIS diestrus females had less center

355 distance than proestrus $(p=0.0016)$ and metestrus $(p=0.0094)$ females (Figure $3 C)$. Within the

356 CNTRL group, estrus females traveled less than proestrus $(p=0.045)$ or metestrus $(0.0130)$

357 females, with diestrus females also traveling less than metestrus $(p=0.023)$ and proestrus

$358\left(p=0.022\right.$; larger panel Figure 3C). Similarly, in the LDT, significant estrous cycle $\left(F_{(3,}\right.$

$35939)=10.43, p<0.0001)$ and SIS $\left(F_{(1,39)}=7.68, p=0.0085\right)$ effects were observed, with SIS estrus

360 females traveling less in the light than proestrus $(p=0.021)$ and metestrus $(p=0.003)$ females.

361 SIS diestrus females also traveled less than metestrus $(p=0.011)$ females (larger panel Figure

362 3D). Although significant SIS effects were observed in the $\operatorname{EPM}\left(F_{(1,39)}=5.87, p=0.0196\right.$;

363 Figure $3 \mathrm{E})$ and FST $\left(F_{(1,17)}=4.47, p=0.04\right.$; Figure $\left.3 \mathrm{~F}\right)$, no estrous cycle effects were found

364 (EPM: $p=0.849$; FST: $p=0.97)$. Within the OF, LDT, EPM, and FST Bonferroni corrected post-

365 hoc comparisons did not reveal differences within estrous states (CNTRLvsSIS; Figures 3C-F

366 smaller panels). Lastly, there was a significant difference in Kaplan Meier survival curves of

367 latency to eat across estrous stages within the SIS group $\left(\chi^{2}(3)=11.71, p=0.0084\right)$, with SIS

368 estrus females having a higher latency to feed than SIS metestrus females $\left(\chi_{(1)}^{2}=15.7\right.$,

$369 \quad p<0.0001$; Figure 3G). Separate Kaplan Meier survival analyses showed differences in latency

370 to eat between CNTRL and SIS females existed across estrous cycle stages $\left(\chi^{2}(8)=32.09\right.$, 


\section{SOCIAL INSTABLITY}

$371 p<0.0001)$, with SIS increasing latency to eat in the proestrus $\left(\chi_{(1)}^{2}=6.91, p=0.0085\right)$, estrus

$372\left(\chi^{2}{ }_{(1)}=14.93, p=0.0001\right)$, and diestrus $\left(\chi_{(1)}^{2}=10.9, p=0.0001\right)$ stages (Figure 3G small panel,

$373 \quad 3 \mathrm{H}-\mathrm{J})$. These results suggest that although the estrous cycle does increase negative valence

374 behaviors during specific stages (mainly estrus and diestrus) the effects of SIS were still

375 observed throughout the estrous cycle. Therefore, variability in behavior across estrous in

376 freely cycling females does not impact the effects of SIS on behavior.

\section{SIS Stress Increases Exogenous Corticosterone Levels in Males and Females}

We next sought to determine whether SIS led to HPA axis activation in males and

380 females. To this end, blood was collected from mice (SIS or CNTRL) at three different time

381 points: prior to SIS exposure, 40 minutes following a SIS cage change (during last week of SIS exposure), and 40 minutes following EPM behavior (Figure 4A). Plasma was then isolated and

$384 \mathrm{x}$ stress $\mathrm{x}$ time of blood collection) since group sizes were equivalent and found no main effect of sex $(p=0.48)$. This result suggests that SIS may be impacting HPA axis activation similarly in males and females. Next, 2x3 repeated measures ANOVA (stress $\mathrm{x}$ time of blood collection)

387 within each sex were performed. In males, we found significant effects of stress $\left(F_{(1,8)}=31.48\right.$, $388 p=0.0005)$ and time of collection $\left(F_{(2,16)}=4.11, p=0.036\right)$ and an interaction $\left(F_{(2,16)}=8.55\right.$,

$389 p=0.003)$. Specifically, SIS males had higher plasma corticosterone levels in response to a cage 390 change $(p=0.0054)$ and EPM $(p<0.0001)$ than CNTRL males (Figure 4B). Significant increases

391 in SIS male plasma corticosterone levels after cage change $(p=0.008)$ and EPM exposure $392(p=0.0001)$ were also observed relative to levels before SIS exposure (Figure 4B). Therefore, 393 chronic SIS exposure increased HPA axis activation in response to acute stressors in males. 


\section{SOCIAL INSTABLITY}

Separate $2 \times 3$ repeated measures ANOVAs in females revealed significant effects of stress $\left(F_{(1,8)}=10, p=0.013\right)$ and time of collection $\left(F_{(2,16)}=5.75, p=0.013\right)$ and an interaction $\left(F_{(2,}\right.$ $\left.{ }_{16}\right)=5.52, p=0.015$ ). SIS females had significantly higher plasma corticosterone levels in response to a cage change than CNTRL females ( $p=0.0007$; Figure $4 \mathrm{C}$ ). Lastly, plasma corticosterone levels in SIS females were significantly higher following a cage change $(p=0.0037)$ and EPM exposure $(p=0.0105)$ as compared to corticosterone levels before SIS exposure (Figure 4C). Thus, similar to males, chronic SIS exposure increased the HPA axis response to acute stressors

401 in females. Taken together, these results demonstrate that SIS leads to HPA axis activation in response to acute stressors in both males and females.

\section{SIS Stress and FLX Impact Adult Hippocampal Neurogenesis}

406 adult hippocampal neurogenesis (44-46) and antidepressants are well-known to increase all

407 stages of adult hippocampal neurogenesis $(13,47-49)$. Therefore, following behavior, mice were

408 perfused, and brains were collected and sectioned for immunostaining. To analyze different

409 stages of adult hippocampal neurogenesis, we immunostained for Ki67 (a proliferative marker;

410 Figure 5A) and DCX (a newborn/immature neuron marker; Figure 5C, 6E). First, we

411 investigated sex differences between groups (CNTRL+VEH, CNTRL+FLX, SIS+VEH, and

412 SIS+FLX) with 2x4 ANOVAs for each hippocampal neurogenesis marker. No effects of sex

413 were found for number of $\mathrm{Ki}^{+}$cells $(p=0.29), \mathrm{DCX}^{+}$cells $(p=0.45), \mathrm{DCX}^{+}$cells with tertiary

414 dendrites $(p=0.093)$, and maturation index $(p=0.086$; Figure 5B and Figure 5D, 6F-G smaller

415 panels). Therefore, separate $2 \times 2$ ANOVAs within each sex were used to investigate SIS and FLX 416 effects on hippocampal neurogenesis. 


\section{SOCIAL INSTABLITY}

Within males, $2 \times 2$ ANOVAs revealed a significant FLX effect $\left(F_{(1,20)}=23.52, p<0.0001\right)$

418 on Ki67 expression, with FLX males having higher number of Ki67+ cells within the dentate

419 gyrus (DG) than VEH treated males [CNTRL+VEHvsCNTRL+FLX $(p=0.0150)$ and

420 SIS+FLXvsSIS+VEH $(p=0.0014)$; Figure 5A-B]. Significant FLX effects were also observed in

421 number of $\mathrm{DCX}^{+}$cells $\left(F_{(1,18)}=30.74, p<0.0001\right)$; DCX cells maturation $\left(F_{(1,18)}=44.1, p<0.0001\right)$;

422 and maturation index $\left(\mathrm{DCX}^{+}\right.$cells with tertiary dendrites/total $\mathrm{DCX}^{+}$cells; $\left(F_{(1,19)}=36.71\right.$,

$423 p=0.0034)$, with FLX treated males having more DCX+ cells [CNTRL+FLXvsCNTRL+VEH

$424(p=0.0067)$ and SIS+FLXvsSIS+VEH $(p=0.0004)$; Figure 5D], more $\mathrm{DCX}^{+}$cells with tertiary

425 dendrites than their VEH male counterparts [CNTRL+FLXvsCNTRL+VEH $(p=0.0004)$ and

426 SIS+FLX $v s$ SIS +VEH $(p=0.0003)$; Figure 5F], and a higher maturation index

427 (SIS+FLXvsSIS+VEH ( $p=0.0401$; Figure 5G) than VEH males. Thus, in males, while FLX

428 increased adult hippocampal neurogenesis, SIS stress did not have significant effects.

429 By contrast, in females separate 2 x2ANOVAs revealed a significant FLX effect $\left(F_{(1,18)}=\right.$

$43055.42, p<0.0001)$ and interaction $\left(F_{(1,18)}=9.968, p=0.0055\right)$ on number of DG Ki67+ cells.

431 Specifically, SIS+VEH females had less Ki67 ${ }^{+}$cells than SIS+FLX $(p<0.0001)$ and

432 CNTRL+VEH $(p=0.0475)$ females (Figure 5B). CNTRL+FLX females also had more Ki67

433 cells than CNTRL+VEH $(p=0.0144)$ females (Figure 5B). Significant effects of SIS $\left(F_{(1,18)}=\right.$

$4345.34, p=0.033)$ and $\operatorname{FLX}\left(F_{(1,18)}=54.79, p<0.0001\right)$, and an interaction $\left(F_{(1,18)}=5.12, p=0.036\right)$

435 was also observed in number of DCX + cells, with SIS+VEH females having less $\mathrm{DCX}^{+}$cells

436 than CNTRL+VEH $(p=0.0065)$ and SIS+FLX $(p<0.0001)$ females. CNTRL+FLX females also

437 had more $\mathrm{DCX}^{+}$cells than $\mathrm{CNTRL}+\mathrm{VEH}$ females $(p=0.0038$; Figure 5D). Further, we observed

438 a significant interaction $\left(F_{(1,18)}=9.14, p=0.0073\right)$ and $\operatorname{FLX}$ effect $\left(F_{(1,18)}=36.01, p<0.0001\right)$ on

439 DCX maturation, with SIS+FLX females having more $\mathrm{DCX}^{+}$cells with tertiary dendrites than 


\section{SOCIAL INSTABLITY}

440 SIS+VEH females $(p<0.0001$; Figure 5F). Lastly, a significant interaction was revealed in

441 maturation index $\left(F_{(1,17)}=9.924, p=0.0058\right)$, with SIS +VEH females having a lower maturation

442 index than SIS+FLX $(p=0.0064)$ and CNTRL+VEH $(p=0.0482)$ females (Figure 5G). Thus,

443 these results show that SIS stress in females does impact proliferation and differentiation.

\section{4}


SOCIAL INSTABLITY

\section{DISCUSSION}

446 Here we demonstrate that the SIS paradigm effectively induces negative valence

447 behaviors and HPA axis activation in both adult males and adult females of the widely used

448 C57BL6/J strain. Importantly, there were no sex differences across all behavior tests following

449 SIS exposure. These data suggest that the SIS paradigm can be used to assess the effects of

450 chronic stress in both sexes without infringing upon ethological validity. We also found that

451 there were sex differences following CORT administration, suggesting that the CORT paradigm

452 to mimic chronic stress is not useful for both sexes. Importantly, we also found that while there

453 were differences in the behavioral effects of SIS among the distinct phases of the estrous cycle,

454 this variability did not result in any significant differences across sex when the entire cohort of

455 females was collapsed. Therefore, SIS can be performed in adult males and freely cycling adult

456 females without concern of estrous cycle confounds.

457 Our study exposes adult male and adult female C57BL/6J mice to a SIS paradigm. The

458 most similar approaches were performed in the outbred CD-1 strain, where adolescent mice

459 exposed to SIS displayed increased negative valence behaviors in EPM and NSF in males (37)

460 and females (36). In these two studies, the CD-1 mice were exposed to SIS beginning at

461 postnatal day 24, which was soon after weaning. Given that anxiety disorders can be heavily

462 influenced by development exposure to stress (50), exposure of adolescent CD-1 mice to SIS

463 may be similar to an early life stress paradigm. In rats, repeated resident-intruder stressful

464 exposure of adolescent females to lactating adult females produces different patterns of effects in

465 negative valence behaviors than exposure of adult females (51). Therefore, exposure of

466 adolescents or adults to SIS may yield different effects. We utilized adult males and adult

467 females from the widely used inbred C57BL/6J strain, and our data supports the notion that adult 


\section{SOCIAL INSTABLITY}

468 exposure to chronic stress can also impact negative valence behaviors, HPA axis activation, and 469 adult hippocampal neurogenesis. In addition, we simultaneously ran the male and female

470 cohorts, allowing direct comparison of potential sex differences. Furthermore, we demonstrate

471 that subsequent FLX treatment reverses the negative valence behaviors in both males and

472 females, lending pharmacological validity to the SIS paradigm as well. By comparing groups

473 (CNTRL+VEH, CNTRL+FLX, SIS+VEH, and SIS+FLX) across sex, we found no sex

474 differences of SIS exposure or FLX treatment in negative valence behaviors or adult

475 hippocampal neurogenesis. Furthermore, there were no sex differences of SIS exposure on HPA

476 axis activation following cage changes or EPM. Additionally, these behavioral results were

477 validated in two separate smaller cohorts of mice, before collapsing the data across both cohorts

478 (data not shown).

479 Subchronic social stress paradigms ( $\sim 15$ days $)$ involving periods of social isolation and

480 crowding have also been assessed in rats. One study found that exposure of adolescent male rats,

481 but not female rats, to social stress induced negative valence behaviors in EPM and HPA axis

482 activation (52). Another study found effects of social stress in adult female rats in inducing

483 negative valence behaviors in EPM (53). Yet another set of studies found that social stress is

484 effective in inducing HPA axis activation in female rats, but defeat is more effective for male

485 rats $(54,55)$. Finally, chronic social stress (4 weeks) combining periods of social isolation and

486 crowding in adult female rats leads to activation of the HPA axis and a decrease in sucrose

487 preference (56). Social stress involving periods of social isolation and crowding also leads to

488 activation of the HPA axis but no effects in EPM or center measures of the OF in adult female

489 CD-1 mice (57). 


\section{SOCIAL INSTABLITY}

Given that ovarian hormones can impact stress responses and behavior $(6,58,59)$ we

491 tracked the estrous cycle throughout our behavioral experiments. We found that SIS estrus and

492 diestrus females travel less distance in the center of the OF and in the light compartment of the

493 LDT than SIS proestrus and metestrus females. These data are in line with findings that socially

494 isolated female mice in the estrus and diestrus phases spend less time in the center of the open

495 field arena than proestrus mice (6). Although behavioral differences across estrous phases were

496 observed in both SIS and CNTRL mice, these differences did not seem to impact our results

497 since stress effects remained when we collapsed female mice from all four stages into one group.

498 The notion that tracking females across all stages of the estrous cycle is necessary when

499 analyzing results is unwarranted because males are just as variable as freely cycling females $(9$,

500 10). Thus, future studies employing the SIS paradigm do not need to track estrous cycle during

501 behavioral experiments.

502 Sex differences in HPA activation between males and females have been found in

503 response to acute stressors (60-62). However, following SIS exposure, we found no sex

504 differences in plasma corticosterone levels in response to either cage changes or EPM exposure.

505 Both males and females exposed to SIS have an increase in plasma corticosterone levels

506 following these acute stressors. Furthermore, prior to SIS exposure, there were no differences

507 between males and females in plasma corticosterone. Future studies may want to assess the long-

508 term neuroendocrine effects of SIS, by analyzing either corticosterone levels several weeks after

509 the final exposure to unstable social environments.

510 Chronic stress can affect endogenous adult hippocampal neurogenesis in male rodents

511 (44-46). Furthermore, chronic treatment with antidepressants, such as the SSRI FLX, increases

512 all stages of adult hippocampal neurogenesis in male and female rodents $(13,47-49)$. To our 


\section{SOCIAL INSTABLITY}

513 knowledge, this is the first study to assess the effects of chronic stress and subsequent

514 antidepressant treatment in both male and female mice simultaneously. Within females, our data

515 shows that SIS impacts multiple stages of adult hippocampal neurogenesis, including

516 proliferation and differentiation. Chronic FLX treatment had effects on all stages of adult

517 neurogenesis in females. By contrast, in males, we did not see any effects of SIS on adult

518 hippocampal neurogenesis but found effects of FLX treatment on proliferation, differentiation,

519 and maturation. In other chronic stress paradigms, male mice administered CORT only displayed

520 an effect on proliferation (13), while male mice exposed to CSDS had transient effects of stress

521 on cell proliferation (63) and a reduction in total $\mathrm{DCX}^{+}$cells (45). However, complete ablation

522 of the hippocampal adult neurogenic niche using focal irradiation does not impact negative

523 valence behaviors, suggesting that decreases in adult hippocampal neurogenesis are not sufficient

524 or necessary to impact behavior (49). Rather, adult neurogenesis is required for the behavioral

525 effects of antidepressant treatment. We found that FLX increased all stages of adult hippocampal

526 neurogenesis in both males and females.

527 Our data suggest that the SIS paradigm is an ethologically valid approach that effectively

528 induces chronic stress in both males and females. In contrast to another chronic stress paradigm,

529 chronic CORT administration, we found no sex differences in the effects of SIS on negative

530 valence behaviors and HPA axis activation. Future work is necessary to determine how long-

531 lasting the effects of SIS are and whether SIS can be leveraged to study stress resilience and

532 susceptibility in both males and females. 
SOCIAL INSTABLITY

\section{FUNDING AND DISCLOSURE}

534 This work was supported by NIMH (R01MH112861, BAS). The authors declare no conflicts of 535 interest.

536

537 ACKNOWLEDGEMENTS

538 The authors would like to thank Emma Diethorn and Sophie Shifman for assistance.

\section{AUTHOR CONTRIBUTIONS}

541 C.N.Y. and B.A.S. conceived of experiments. C.N.Y., S.A.A., L.B., and A.G. performed the

542 experiments. C.N.Y., M.M.G., and B.A.S. analyzed the data and made the figures. C.N.Y.,

543 M.M.G., and B.A.S. wrote the manuscript. 


\section{SOCIAL INSTABLITY}

\section{6}

547

548

549

550

551

552

553

554

555

556

557

558

559

560

561

562

563

564

565

566

567

568

\section{REFERENCES}

1. Beery, A. K., \& Zucker, I. (2011). Sex bias in neuroscience and biomedical research. Neurosci Biobehav Rev, 35(3), 565-572.

2. Autry AE, Adachi M, Cheng P, Monteggia LM (2009). Genderspecific impact of brainderived neurotrophic factor signaling on stress-induced depression-like behavior. Biol Psychiatry 66: 84-90.

3. Trainor, B.C. (2011). Stress responses and the mesolimbic dopamine system: social contexts and sex differences. Horm Behav 60: 457-469.

4. Shansky RM (2015). Sex differences in PTSD resilience and susceptibility: challenges for animal models of fear learning. Neurobiol Stress 1: 60-65.

5. Becker, J. B., Arnold, A. P., Berkley, K. J., Blaustein, J. D., Eckel, L. A., Hampson, E., ... \& Taylor, J. (2005). Strategies and methods for research on sex differences in brain and behavior. Endocrinology, 146(4), 1650-1673.

6. Palanza, P., Gioiosa, L., \& Parmigiani, S. (2001). Social stress in mice: gender differences and effects of estrous cycle and social dominance. Physiol Behav, 73(3), 411420.

7. Shansky, R. M., \& Woolley, C. S. (2016). Considering sex as a biological variable will be valuable for neuroscience research. J Neurosci, 36(47), 11817-11822.

8. Yohn, C. N., Shifman, S., Garino, A., Diethorn, E., Bokka, L., Ashamalla, S. A., \& Samuels, B. A. (2018). Fluoxetine effects on behavior and adult hippocampal neurogenesis in female C57BL/6J mice across the estrous cycle. bioRxiv, 368449.

9. Becker, J. B., Prendergast, B. J., \& Liang, J. W. (2016). Female rats are not more variable than male rats: a meta-analysis of neuroscience studies. Biol Sex Differ, 7(1), 34. 


\section{SOCIAL INSTABLITY}

10. Prendergast, B. J., Onishi, K. G., \& Zucker, I. (2014). Female mice liberated for inclusion in neuroscience and biomedical research. Neurosci Biobehav Rev, 40, 1-5.

11. Shansky, R. M. (2018). Sex differences in behavioral strategies: avoiding interpretational pitfalls. Current opinion in neurobiology, 49, 95-98.

12. Blanchard, D. C., Griebel, G., \& Blanchard, R. J. (1995). Gender bias in the preclinical psychopharmacology of anxiety: male models for (predominantly) female disorders. $J$ Psychopharmacol, 9(2), 79-82.

13. David, D. J., Samuels, B. A., Rainer, Q., Wang, J. W., Marsteller, D., Mendez, I., ... \& Artymyshyn, R. P. (2009). Neurogenesis-dependent and-independent effects of fluoxetine in an animal model of anxiety/depression. Neuron, 62(4), 479-493.

14. Golden, S. A., Covington III, H. E., Berton, O., \& Russo, S. J. (2011). A standardized protocol for repeated social defeat stress in mice. Nat Protoc, 6(8), 1183.

15. Russo, S. J., \& Nestler, E. J. (2013). The brain reward circuitry in mood disorders. Nat Rev Neurosci, 14(9), 609.

16. Mineur, Y. S., Belzung, C., \& Crusio, W. E. (2006). Effects of unpredictable chronic mild stress on anxiety and depression-like behavior in mice. Behav Brain Res, 175(1), 4350.

17. Piantadosi, S. C., French, B. J., Poe, M. M., Timić, T., Marković, B. D., Pabba, M., ... \& Cook, J. M. (2016). Sex-dependent anti-stress effect of an $\alpha 5$ subunit containing GABAA receptor positive allosteric modulator. Front Pharmacol, 7, 446.

18. Guilloux, J. P., Seney, M., Edgar, N., \& Sibille, E. (2011). Integrated behavioral zscoring increases the sensitivity and reliability of behavioral phenotyping in mice: relevance to emotionality and sex. J Neurosci Meth, 197(1), 21-31. 


\section{SOCIAL INSTABLITY}

19. Zhao, Z., Zhang, L., Guo, X. D., Cao, L. L., Xue, T. F., Zhao, X. J., ... \& Sun, X. L. (2017). Rosiglitazone exerts an anti-depressive effect in unpredictable chronic mildstress-induced depressive mice by maintaining essential neuron autophagy and inhibiting excessive astrocytic apoptosis. Front Mol Neurosci, 10, 293.

20. Belzung, C., \& Lemoine, M. (2011). Criteria of validity for animal models of psychiatric disorders: focus on anxiety disorders and depression. Biology of mood \& anxiety disorders, 1(1), 9.

21. Farooq, R. K., Isingrini, E., Tanti, A., Le Guisquet, A. M., Arlicot, N., Minier, F., ... \& Camus, V. (2012). Is unpredictable chronic mild stress (UCMS) a reliable model to study depression-induced neuroinflammation?. Behav Brain Res, 231(1), 130-137.

22. Willner, P. (1997). Validity, reliability and utility of the chronic mild stress model of depression: a 10-year review and evaluation. Psychopharmacology, 134(4), 319-329.

23. Krishnan, V., Han, M. H., Graham, D. L., Berton, O., Renthal, W., Russo, S. J., ... \& Ghose, S. (2007). Molecular adaptations underlying susceptibility and resistance to social defeat in brain reward regions. Cell, 131(2), 391-404

24. Keeney, A., Jessop, D. S., Harbuz, M. S., Marsden, C. A., Hogg, S., \& Blackburn-Munro, R. E. (2006). Differential effects of acute and chronic social defeat stress on hypothalamic-pituitary-adrenal axis function and hippocampal serotonin release in mice. J Neuroendocrinol, 18(5), 330-338.

25. Greenberg, G. D., Laman-Maharg, A., Campi, K. L., Voigt, H., Orr, V. N., Schaal, L., \& Trainor, B. C. (2014). Sex differences in stress-induced social withdrawal: role of brain derived neurotrophic factor in the bed nucleus of the stria terminalis. Front Behav Neurosci, 7, 223. 


\section{SOCIAL INSTABLITY}

615 26. Harris, A. Z., Atsak, P., Bretton, Z. H., Holt, E. S., Alam, R., Morton, M. P., ... \&

616 Gordon, J. A. (2018). A novel method for chronic social defeat stress in female

617 mice. Neuropsychopharmacol, 43(6), 1276.

618 27. Takahashi, A., Chung, J. R., Zhang, S., Zhang, H., Grossman, Y., Aleyasin, H., ... \&

619 Hodes, G. E. (2017). Establishment of a repeated social defeat stress model in female 620 mice. Sci Rep, 7(1), 12838.

621 28. Herman, J. P., McKlveen, J. M., Ghosal, S., Kopp, B., Wulsin, A., Makinson, R., ... \&

622 Myers, B. (2016). Regulation of the hypothalamic-pituitary-adrenocortical stress

623 response. Compr Physiol, 6(2), 603

624 29. Kitay, J. I. (1963). Pituitary-adrenal function in the rat after gonadectomy and gonadal

625 hormone replacement. Endocrinology, 73(2), 253-260.

626 30. Wardenaar, K. J., Vreeburg, S. A., van Veen, T., Giltay, E. J., Veen, G., Penninx, B. W.,

627 \& Zitman, F. G. (2011). Dimensions of depression and anxiety and the hypothalamo-

628 pituitary-adrenal axis. Biol Psychiatry, 69(4), 366-373.

629 31. Gourley, S. L., Wu, F. J., \& Taylor, J. R. (2008). Corticosterone regulates pERK1/2 map

$630 \quad$ kinase in a chronic depression model. Ann NY Acad Sci, 1148(1), 509-514.

631 32. Gourley, S. L., \& Taylor, J. R. (2011). Induction of persistent depressive-like behavior by

632 corticosterone. In Mood and Anxiety Related Phenotypes in Mice (pp. 251-265). Humana

$633 \quad$ Press.

634 33. Brummelte, S., \& Galea, L. A. (2010). Chronic corticosterone during pregnancy and 635 postpartum affects maternal care, cell proliferation and depressive-like behavior in the 636 dam. Horm Behav, 58(5), 769-779. 


\section{SOCIAL INSTABLITY}

34. Brummelte, S., Pawluski, J. L., \& Galea, L. A. (2006). High post-partum levels of corticosterone given to dams influence postnatal hippocampal cell proliferation and behavior of offspring: a model of post-partum stress and possible depression. Horm Behav, 50(3), 370-382.

35. Mekiri, M., Gardier, A. M., David, D. J., \& Guilloux, J. P. (2017). Chronic corticosterone administration effects on behavioral emotionality in female c57bl6 mice. Exp Clin Psychopharmacol, 25(2), 94.

36. Schmidt, M. V., Scharf, S. H., Liebl, C., Harbich, D., Mayer, B., Holsboer, F., \& Müller, M. B. (2010). A novel chronic social stress paradigm in female mice. Horm Behav, 57(45), 415-420.

37. Sterlemann, V., Ganea, K., Liebl, C., Harbich, D., Alam, S., Holsboer, F., ... \& Schmidt, M. V. (2008). Long-term behavioral and neuroendocrine alterations following chronic social stress in mice: implications for stress-related disorders. Horm Behav, 53(2), 386394

38. Schmidt, M. V., Sterlemann, V., Ganea, K., Liebl, C., Alam, S., Harbich, D., ... \& Müller, M. B. (2007). Persistent neuroendocrine and behavioral effects of a novel, etiologically relevant mouse paradigm for chronic social stress during adolescence. Psychoneuroendocrinol, 32(5), 417-429.

39. Bartolomucci, A., Pederzani, T., Sacerdote, P., Panerai, A. E., Parmigiani, S., \& Palanza, P. (2004). Behavioral and physiological characterization of male mice under chronic psychosocial stress. Psychoneuroendocrinol, 29(7), 899-910. 


\section{SOCIAL INSTABLITY}

40. McLean, A. C., Valenzuela, N., Fai, S., \& Bennett, S. A. (2012). Performing vaginal lavage, crystal violet staining, and vaginal cytological evaluation for mouse estrous cycle staging identification. Journal of visualized experiments: JoVE, (67).

41. Byers, S. L., Wiles, M. V., Dunn, S. L., \& Taft, R. A. (2012). Mouse estrous cycle identification tool and images. PloS ONE, 7(4), e35538.

42. Felicio, L. S., Nelson, J. F., \& Finch, C. E. (1984). Longitudinal studies of estrous cyclicity in aging C57BL/6J mice: II. Cessation of cyclicity and the duration of persistent vaginal cornification. Biol Reprod, 31(3), 446-453.

43. Franklin, K. B., \& Paxinos, G. (2008). The mouse brain in stereotaxic coordinates, compact. The coronal plates and diagrams. Amsterdam: Elsevier Academic Press.

44. Czéh, B., Welt, T., Fischer, A. K., Erhardt, A., Schmitt, W., Müller, M. B., ... \& Keck, M. E. (2002). Chronic psychosocial stress and concomitant repetitive transcranial magnetic stimulation: effects on stress hormone levels and adult hippocampal neurogenesis. Biol Psychiatry, 52(11), 1057-1065.

45. Van Bokhoven, P., Oomen, C. A., Hoogendijk, W. J. G., Smit, A. B., Lucassen, P. J., \& Spijker, S. (2011). Reduction in hippocampal neurogenesis after social defeat is longlasting and responsive to late antidepressant treatment. Eur J Neurosci, 33(10), 18331840.

46. Pham, K., Nacher, J., Hof, P. R., \& McEwen, B. S. (2003). Repeated restraint stress suppresses neurogenesis and induces biphasic PSA-NCAM expression in the adult rat dentate gyrus. Eur J Neurosci, 17(4), 879-886. 


\section{SOCIAL INSTABLITY}

47. Malberg, J. E., Eisch, A. J., Nestler, E. J., \& Duman, R. S. (2000). Chronic antidepressant treatment increases neurogenesis in adult rat hippocampus. $J$ Neurosci, 20(24), 91049110.

48. Hill, A. S., Sahay, A., \& Hen, R. (2015). Increasing adult hippocampal neurogenesis is sufficient to reduce anxiety and depression-like behaviors. Neuropsychopharmacol, 40(10), 2368.

49. Santarelli, L., Saxe, M., Gross, C., Surget, A., Battaglia, F., Dulawa, S., ... \& Belzung, C. (2003). Requirement of hippocampal neurogenesis for the behavioral effects of antidepressants. Science, 301(5634), 805-809.

50. Leonardo, E. D., \& Hen, R. (2008) Anxiety and developmental disorders. Neuropsychopharm, 33 (1), 134.

51. Ver Hoeve, E. S., Kelly, G., Luz, S., Ghanshani, S., \& Bhatnagar, S. (2013). Short-term and long-term effects of repeated social defeat during adolescence or adulthood in female rats. Neuroscience, 249, 63-73.

52. Roeckner, A. R., Bowling, A., \& Butler, T. R. (2017). Chronic social instability increases anxiety-like behavior and ethanol preference in male Long Evans rats. Physiol Behav, 173, 179-187.

53. Haller, J., Leveleki, C., Baranyi, J., Mikics, E., \& Bakos, N. (2003). Stress, social avoidance and anxiolytics: a potential model of stress-induced anxiety. Behav Pharmacol, 14(5), 439-446.

54. Haller, J., Fuchs, E., Halász, J., \& Makara, G. B. (1999). Defeat is a major stressor in males while social instability is stressful mainly in females: towards the development of a social stress model in female rats. Brain Res Bull, 50(1), 33-39. 


\section{SOCIAL INSTABLITY}

55. Nowacka, M. M., Paul-Samojedny, M., Bielecka, A. M., Plewka, D., Czekaj, P., \& Obuchowicz, E. (2015). LPS reduces BDNF and VEGF expression in the structures of the HPA axis of chronic social stressed female rats. Neuropeptides, 54, 17-27.

56. Herzog, C. J., Czéh, B., Corbach, S., Wuttke, W., Schulte-Herbrüggen, O., Hellweg, R., ... \& Fuchs, E. (2009). Chronic social instability stress in female rats: a potential animal model for female depression. Neuroscience, 159(3), 982-992.

57. Jarcho, M. R., Massner, K. J., Eggert, A. R., \& Wichelt, E. L. (2016). Behavioral and physiological response to onset and termination of social instability in female mice. Horm Behav, 78, 135-140.

58. Sisk, C. L., \& Zehr, J. L. (2005). Pubertal hormones organize the adolescent brain and behavior. Front Neuroendocrinol, 26(3-4), 163-174

59. Wood, G. E., Beylin, A. V., \& Shors, T. J. (2001). The contribution of adrenal and reproductive hormones to the opposing effects of stress on trace conditioning males versus females. Behav Neurosci, 115(1), 175.

60. Kirschbaum, C., Kudielka, B. M., Gaab, J., Schommer, N. C., \& Hellhammer, D. H. (1999). Impact of gender, menstrual cycle phase, and oral contraceptives on the activity of the hypothalamus-pituitary-adrenal axis. Psychosom Med, 61(2), 154-162.

61. Kirschbaum, C., Wüst, S., \& Hellhammer, D. (1992). Consistent sex differences in cortisol responses to psychological stress. Psychosom Med, 54(6), 648-657.

62. Kudielka, B. M., \& Kirschbaum, C. (2005). Sex differences in HPA axis responses to stress: a review. Biol Psychology, 69(1), 113-132.

63. Lagace, D. C., Donovan, M. H., DeCarolis, N. A., Farnbauch, L. A., Malhotra, S., Berton, O., ... \& Eisch, A. J. (2010). Adult hippocampal neurogenesis is functionally 
bioRxiv preprint doi: https://doi.org/10.1101/550525; this version posted February 14,2019 . The copyright holder for this preprint (which was not certified by peer review) is the author/funder. All rights reserved. No reuse allowed without permission.

\section{SOCIAL INSTABLITY}


SOCIAL INSTABLITY

\section{FIGURE LEGENDS}

730 Figure 1 Behavioral Effects of Chronic Corticosterone Administration in Male and Female

731 Mice. (A) Timeline of chronic CORT paradigm and keys for larger graphs. (B-E) Small panels

732 represent 2x4 ANOVAs Bonferroni posthocs of Sex $x$ Group (VEH+VEH, VEH+FLX,

733 CORT+VEH, CORT+FLX) with sex differences observed in distance traveled in light (C small

734 panel: $F_{(1,72)}=13.69, p<0.001, * * \mathrm{CORT}+\mathrm{VEH}$ males $v s \mathrm{CORT}+\mathrm{VEH}$ females $\left.p=0.001\right)$ and on

735 open arms of EPM (D small panel: $F_{(1,72)}=9.97, p<0.001, *$ CORT+VEH males $v s$ CORT + VEH

736 females $p=0.005$ ). Larger (B-E) panels represent separate $2 \times 2$ ANOVAs Bonferroni posthocs

737 (CORT $x$ FLX) within each sex. Differences in males were observed in LDT (CORT:

$738 F_{(1,36)}=13.53, p=0.0008 ; \mathrm{FLX}: F_{(1,36)}=9.88, p=0.0033 ; * * \mathrm{CORT}+\mathrm{VEH} v s \mathrm{VEH}+\mathrm{VEH} p=0.0007$

739 and $*$ CORT+VEHvsCORT+FLX $(p=0.0017)$, EPM open arm distance $\left(\right.$ FLX: $F_{(1,36)}=25.68$,

$740 \quad p<0.0001$, INTERACTION: $F_{(1,36)}=5.29, p=0.027 ; * \mathrm{CORT}+\mathrm{VEH} v s \mathrm{VEH}+\mathrm{VEH} p=0.025$ and

$741 * * \mathrm{CORT}+\mathrm{VEH} v s \mathrm{CORT}+\mathrm{FLX} p=0.0042)$, and FST immobility $\left(\mathrm{FLX}: F_{(1,36)}=39.38, p<0.00001\right.$;

$742 * * * \mathrm{VEH}+\mathrm{VEH} v \mathrm{VEH}+\mathrm{FLX} p<0.0001, * * * * \mathrm{CORT}+\mathrm{VEH} v \mathrm{CORT}+\mathrm{FLX} p<0.0001)$. Smaller

743 scatterplot (F) represents sex differences observed by Kaplan Meier survival analysis (G)

$744 * * *$ CORT+VEH males $v s$ CORT+VEH females $p<0.001$. Larger scatterplot (F) shows NSF data

745 showing individual latency to eat values with Kaplan Meier survival analysis in males $(\mathrm{H})$

$746 * * * * \mathrm{CORT}+\mathrm{VEH} v \mathrm{VEH}+\mathrm{VEH} p<0.0001 ; * * * * \mathrm{CORT}+\mathrm{VEH} v s \mathrm{CORT}+\mathrm{FLX}, p<0.0001)$.

747 Additionally, differences in female detected in EPM open arm distance (D; FLX: $F_{(1,36)}=5.88$,

$748 p=0.02 ; * \mathrm{VEH}+\mathrm{VEH} v \mathrm{VEH}+\mathrm{FLX} p=0.018)$ and FST immobility $\left(\mathrm{E} ; \mathrm{FLX}: F_{(1,35)}=28.37\right.$,

$\left.749 p<0.0001, * * * * \mathrm{VEH}+\mathrm{VEH} v \mathrm{VEH}+\mathrm{FLX} \mathrm{p}<0.0001,{ }^{*} \mathrm{CORT}+\mathrm{VEH} v s \mathrm{CORT}+\mathrm{FLX} p=0.018\right)$.

750 Scatterplot (F) represents feeding behavior differences form Kaplan Meier survival analysis

751 between $* \mathrm{VEH}+\mathrm{VEH}$ and $\mathrm{VEH}+\mathrm{FLX}(p=0.033)$. 
SOCIAL INSTABLITY

753 Figure 2 Behavioral Effects of Social Instability Stress (SIS) in Male and Female Mice. (A)

754 Timeline of SIS paradigm and keys for larger graphs. (B-E) Small panels represent 2x4

755 ANOVAs Bonferroni posthocs of Sex $x$ Groups (CNTRL+VEH, CNTRL+FLX, SIS+VEH,

756 SIS+FLX) with no sex differences observed in any behavior. Larger (B-E) panels represent

757 separate 2x2 ANOVAs Bonferroni posthocs (SISxFLX) within each sex. Differences in males

758 were observed in OF (SIS: $F_{(1,83)}=4.148, p=0.0449$; interaction: $F_{(1,83)}=4.087, p=0.0464$;

$759 * * \mathrm{CNTRL}+\mathrm{VEH} v s \mathrm{SIS}+\mathrm{VEH}, p=0.0041)$ as well as in females; (SIS: $F_{(1,91)}=5.017, p=0.0276$ :

760 SISxFLX: $\left.F_{(1,91)}=4.643, p=0.0339 ; *{ }^{*} \mathrm{CNTRL}+\mathrm{VEH} v \mathrm{SIS}+\mathrm{VEH}, p=0.0019\right)$. LDT $(\mathrm{CORT}$ :

$761 \quad F_{(1,83)}=10.53, p=0.0017$; FLX: $F_{(1,83)}=7.076, p=0.0094 ; * *$ CNTRL+VEHvsSIS+VEH $p=0.029$

762 and $* * *$ SIS+VEHvsSIS+FLX $(p<0.0001)$,EPM open arm distance $\left(\right.$ FLX: $F_{(1,36)}=25.68$,

$763 p<0.0001$, INTERACTION: $F_{(1,36)}=5.29, p=0.027 ; *$ CORT+VEHvsVEH+VEH $p=0.025$ and

$764 * *$ CORT+VEHvsCORT+FLX $p=0.0042)$, and FST immobility $\left(\right.$ FLX: $F_{(1,36)}=39.38, p<0.00001$;

$765 * * * \mathrm{VEH}+\mathrm{VEH} v \mathrm{VEH}+\mathrm{FLX} p<0.0001, * * * * \mathrm{CORT}+\mathrm{VEH} v \mathrm{CCORT}+\mathrm{FLX} p<0.0001)$. Smaller

766 scatterplot (F) represents sex differences observed by Kaplan Meier survival analysis (G)

$767 * * * \mathrm{CORT}+\mathrm{VEH}$ males $v s \mathrm{CORT}+\mathrm{VEH}$ females $p<0.001$. Larger scatterplot $(\mathrm{F})$ shows NSF data

768 showing individual latency to eat values with Kaplan Meier survival analysis in males $(\mathrm{H})$

$769 * * * * \mathrm{CORT}+\mathrm{VEH} v s \mathrm{VEH}+\mathrm{VEH} p<0.0001 ; * * * * \mathrm{CORT}+\mathrm{VEH} v \mathrm{CCORT}+\mathrm{FLX}, p<0.0001)$.

770 Additionally, differences in female detected in EPM open arm distance (D; FLX: $F_{(1,36)}=5.88$,

$771 p=0.02 ; * \mathrm{VEH}+\mathrm{VEH} v \mathrm{VEH}+\mathrm{FLX} p=0.018)$ and FST immobility $\left(\mathrm{E} ; \mathrm{FLX}: F_{(1,35)}=28.37\right.$,

$772 p<0.0001, * * * * \mathrm{VEH}+\mathrm{VEH} v \mathrm{VEH}+\mathrm{FLX} p<0.0001, * \mathrm{CORT}+\mathrm{VEH} v s \mathrm{CORT}+\mathrm{FLX} p=0.018)$.

773 Scatterplot (F) represents feeding behavior differences form Kaplan Meier survival analysis

774 between $* \mathrm{VEH}+\mathrm{VEH}$ and $\mathrm{VEH}+\mathrm{FLX} p=0.033$. 
SOCIAL INSTABLITY

Figure 3. Impact of Estrous Cycle in Females on Behavior. Time line of experiment (A) and

777 representative 10x images of vaginal smears (B). Larger panels (C-F) represent 2x4 ANOVAs

778 (SIS $x$ ESTROUS) in the OF $\left(* * * *\right.$ SIS: $F_{(1,39)}=19.13, p<0.0001$; ESTROUS: $F_{(3,39)}=45.16$,

$779 p<0.001 ; * * *$ SIS+ESTRUSvsSIS+PROESTRUS $p=0.0003$;

$780 * *$ SIS+ESTRUS $v$ SIS+METESTRUS $p=0.013 ; * *$ SIS+DIESTRUSvsSIS+PROESTRUS

$781 \quad p=0.0016 ; * *$ SIS+DIESTRUS $v s S I S+$ METESTRUS $p=0.0094$

$782 * * *$ CNTRL+ESTRUSvsCNTRL+PROESTRUS $p=0.045$

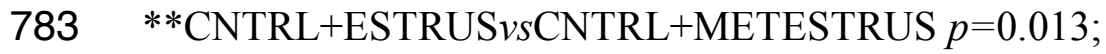

$784 *$ CNTRL+DIESTRUSvsCNTRL+PROESTRUS $p=0.022$;

$785 *$ CNTRL+DIESTRUSvsCNTRL+METESTRUS $p=0.023)$, LDT $\left(* * \operatorname{SIS}: F_{(1,39)}=7.68\right.$,

$786 p=0.0085$; ESTROUS: $F_{(3,39)}=10.43, p<0.0001$ Bonferroni corrected comparisons

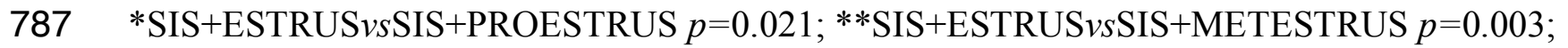

$788 *$ SIS+DIESTRUSvsSIS+METESTRUS $p=0.011)$, EPM $\left(* \operatorname{SIS}: F_{(1,39)}=5.87, p=0.0196\right)$, and FST

789 (*SIS: $\left.F_{(1,17)}=4.47, p=0.04\right)$. No differences within estrous state between SIS and CNTRL were

790 observed smaller panels (C-F). Scatterplot (G) form Kaplan Meier survival analysis of latency to

791 eat across estrous stages within the SIS group $\left(\chi^{2}(3)=11.71, p=0.0084\right.$;

$792 * * * *$ SIS+ESTRUSvsSIS+METESTRUS $p<0.0001)$. Comparison between SIS and CNTRL

793 across estrous cycle (smaller panel G) represent differences in Kaplan Meier survival analysis of

794 latency to eat across **SIS+PROESTRUSvsCNTRL+PROESTRUS $p=0.0085(\mathrm{H})$,

$795 * * *$ SIS+ESTRUSvsCNTRL+ESTRUS $p=0.0001(\mathrm{I})$

$796 * * *$ SIS+DIESTRUSvsCNTRL+DIESTRUS $p=0.0001(\mathrm{~J})$.

797 


\section{SOCIAL INSTABLITY}

799 Figure 4. SIS Stress Increases Exogenous Corticosterone Levels in Males and Females. Timeline

800 of experiment and legend for figures (A). Smaller panels (B-C) illustrate the changes in plasma

801 corticosterone levels across blood collection time points. Larger panel (B) displays 2x3

802 ANOVAs (SIS $x$ TIME) within males with SIS $\left(F_{(1,8)}=31.48, p=0.0005\right)$ and TIME effects $\left(F_{(2,}\right.$

$80316)=4.11, p=0.036 ; *$ SIS + PRIOR $v s S I S+$ CAGE $p=0.008, * * *$ SIS + PRIOR $v s$ SIS + EPM

$804 p=0.0001)$ and INTERACTION $\left(F_{(2,16)}=8.55, p=0.003, * * \mathrm{SIS}+\mathrm{CAGE} v \operatorname{sCNTRL}+\mathrm{CAGE}\right.$

$805 p=0.0054 ; * * * *$ SIS + EPMvsCNTRL+EPM $p<0.0001)$. In females, larger panel (C), SIS $\left(F_{(1,}\right.$

$\left.806{ }_{8}=10, p=0.013\right)$ and TIME effects $\left(F_{(2,16)}=5.75, p=0.013 ; * * \mathrm{SIS}+\mathrm{PRIOR} v s \mathrm{SIS}+\mathrm{CAGE}\right.$

$807 p=0.0037, * * * \operatorname{SIS}+\mathrm{PRIOR} v s \mathrm{SIS}+\mathrm{EPM} p=0.0105)$ and $\operatorname{INTERACTION}\left(F_{(2,16)}=5.52, p=0.015\right.$,

$808 * * * \mathrm{SIS}+\mathrm{CAGEvsCNTRL}+\mathrm{CAGE} p=0.0007)$.

809

810 Figure 5. SIS Stress and FLX Effects on Adult Hippocampal Neurogenesis. Representative 10x

811 images of Ki67 (A) and DCX (C), with 40x images used to quantify DCX ${ }^{+}$cells with tertiary

812 dendrites (E). Smaller panels (B, D, F, G) display no sex differences in adult hippocampal

813 neurogenesis. In males $2 \times 2$ ANOVAs ( $\operatorname{SIS} x \mathrm{FLX})$ revealed FLX effects in $\operatorname{Ki}^{+}\left(F_{(1,20)}=23.52\right.$,

$814 p<0.0001 ; * \mathrm{CNTRL}+\mathrm{VEH} v s \mathrm{CNTRL}+\mathrm{FLX} p=0.0150$ and $* * \mathrm{SIS}+\mathrm{FLX} v s \mathrm{SIS}+\mathrm{VEH} p=0.0014)$,

$815 \operatorname{DCX}^{+}\left(F_{(1,18)}=30.74, p<0.0001 ; * * \mathrm{CNTRL}+\mathrm{FLX} v s \mathrm{CNTRL}+\mathrm{VEH} p=0.0067\right.$ and

$\left.816 * * * \mathrm{SIS}+\mathrm{FLX} v_{s} \mathrm{SIS}+\mathrm{VEH} p=0.0004\right), \mathrm{DCX}^{+}$cells with tertiary dendrites $\left(F_{(1,18)}=44.1, \mathrm{p}<0.0001\right.$;

$817 * * * \mathrm{CNTRL}+\mathrm{FLX} v s \mathrm{CNTRL}+\mathrm{VEH} p=0.0004$ and $* * * \mathrm{SIS}+\mathrm{FLX} v s \mathrm{SIS}+\mathrm{VEH} p=0.0003)$ and

818 maturation index $\left(F_{(1,19)}=36.71, p=0.0034 ; * * \mathrm{SIS}+\mathrm{FLX} v s \mathrm{SIS}+\mathrm{VEH} p=0.0401\right)$. Within females

$8192 \times 2$ ANOVAs (SIS $x$ FLX) showed main effects and interactions in Ki67 (FLX: $F_{(1,18)}=55.42$,

$820 \mathrm{p}<0.0001$; INTERACTION: $F_{(1,18)}=9.968, p=0.0055 ; * * * * \mathrm{SIS}+\mathrm{VEH} v s \mathrm{SIS}+\mathrm{FLX} p<0.0001$,

$821 * \mathrm{SIS}+\mathrm{VEH} v \mathrm{CNTRL}+\mathrm{VEH} p=0.0475, * \mathrm{CNTRL}+\mathrm{FLX} v s \mathrm{CNTRL}+\mathrm{VEH} p=0.014), \mathrm{DCX}$ (SIS: 
SOCIAL INSTABLITY

$822 F_{(1,18)}=5.34, p=0.033$, FLX: $F_{(1,18)}=54.79, p<0.0001$, INTERACTION: $F_{(1,18)}=5.12, p=0.036$;

$823 * * * * \mathrm{SIS}+\mathrm{VEH} v s \mathrm{SIS}+\mathrm{FLX} p<0.0001, * * \mathrm{SIS}+\mathrm{VEH} v \mathrm{CNTRL}+\mathrm{VEH} p=0.0065$,

$824 * * \mathrm{CNTRL}+\mathrm{FLX} v s \mathrm{CNTRL}+\mathrm{VEH} p=0.0038), \mathrm{DCX}^{+}$cells with tertiary dendrites

825 (INTERACTION $F_{(1,18)}=9.14, p=0.0073$, FLX: $F_{(1,18)}=36.01, p<0.0001$;

$826 * * * * \mathrm{SIS}+\mathrm{VEH} v s \mathrm{SIS}+\mathrm{FLX} p<0.0001)$, and maturation index (INTERACTION: $F_{(1,17)}=9.924$,

$\left.827 p=0.0058 ; * * \mathrm{SIS}+\mathrm{VEH} v s \mathrm{SIS}+\mathrm{FLX} p=0.0064,{ }^{*} \mathrm{SIS}+\mathrm{VEH} v \mathrm{CNTRL}+\mathrm{VEH} p=0.048\right)$. 
$A$

- VEH+VEH
- VEH+FLX
- CORT+VEH
- CORT+FLX

B

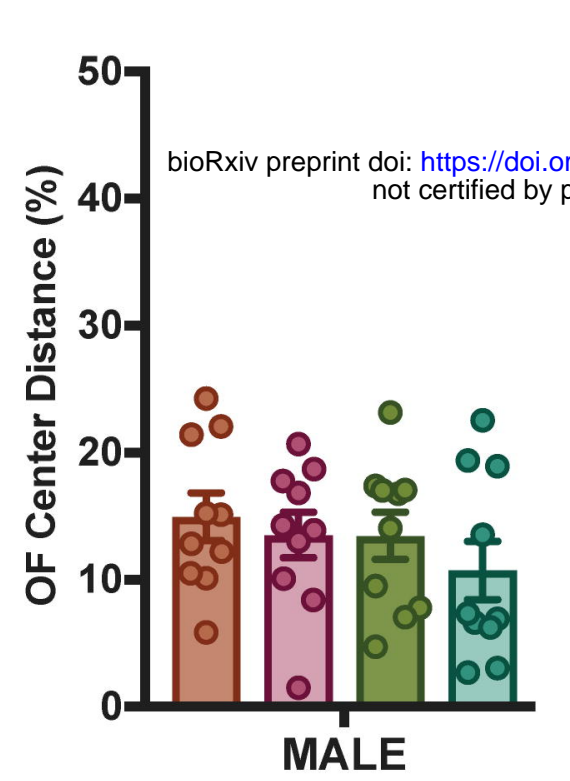

D

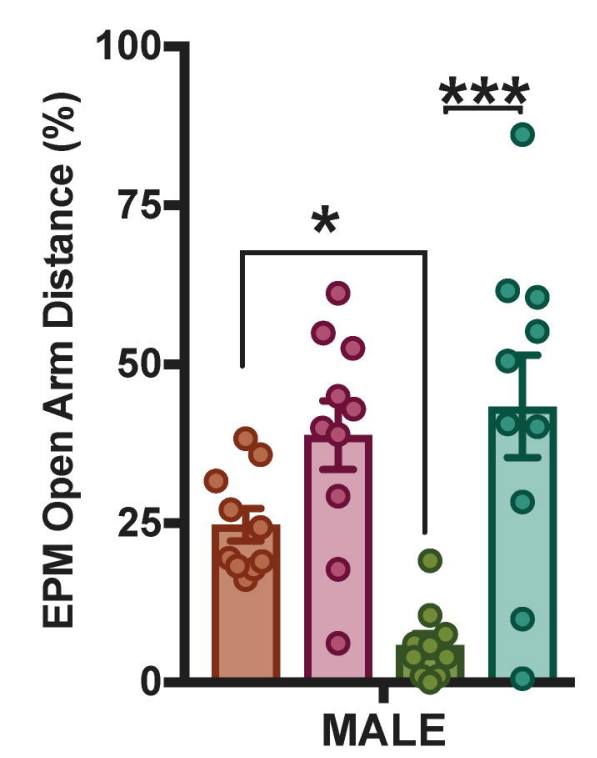

F

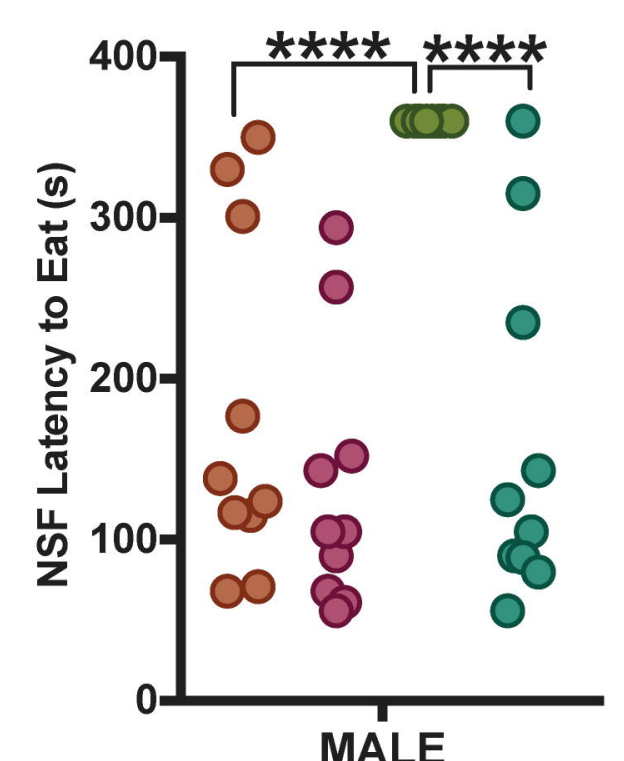

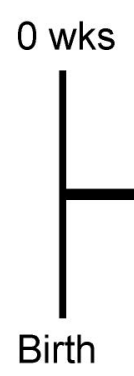

$15-16$ wks

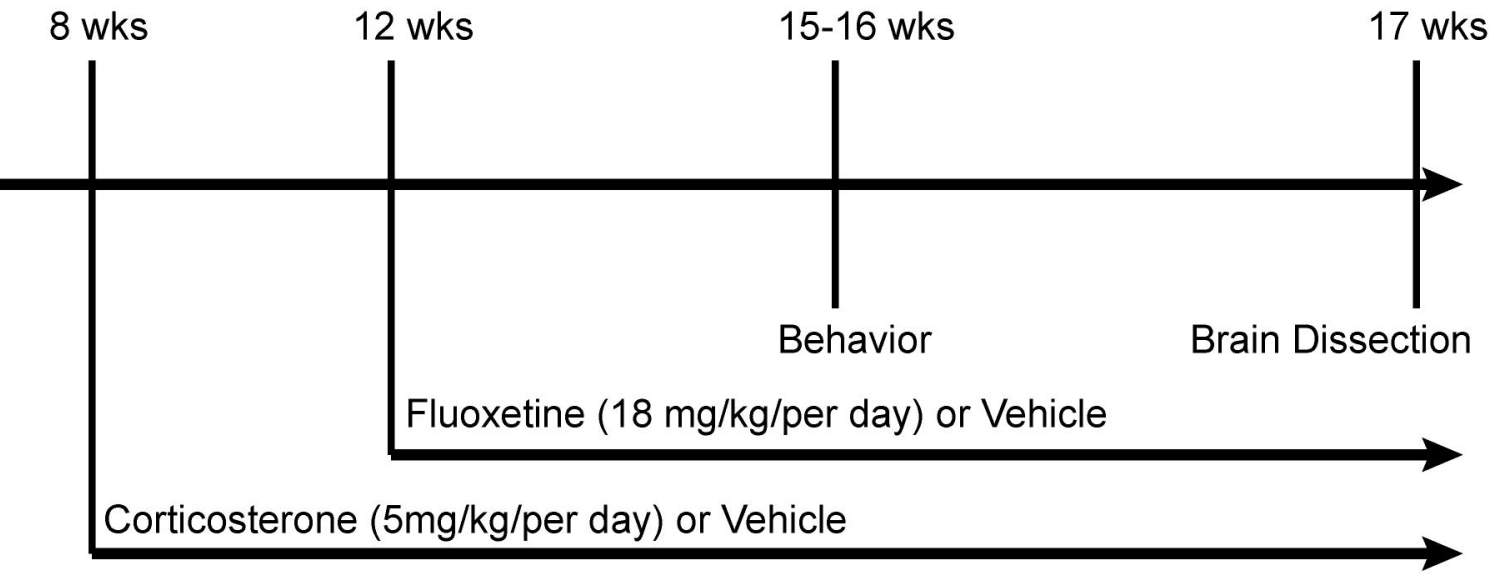

C

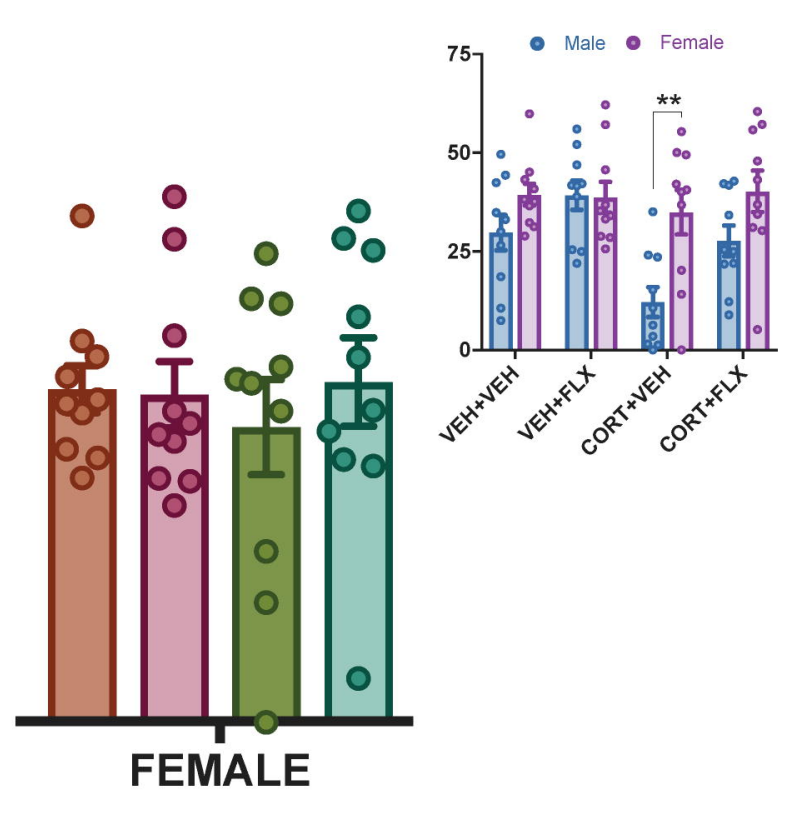

E
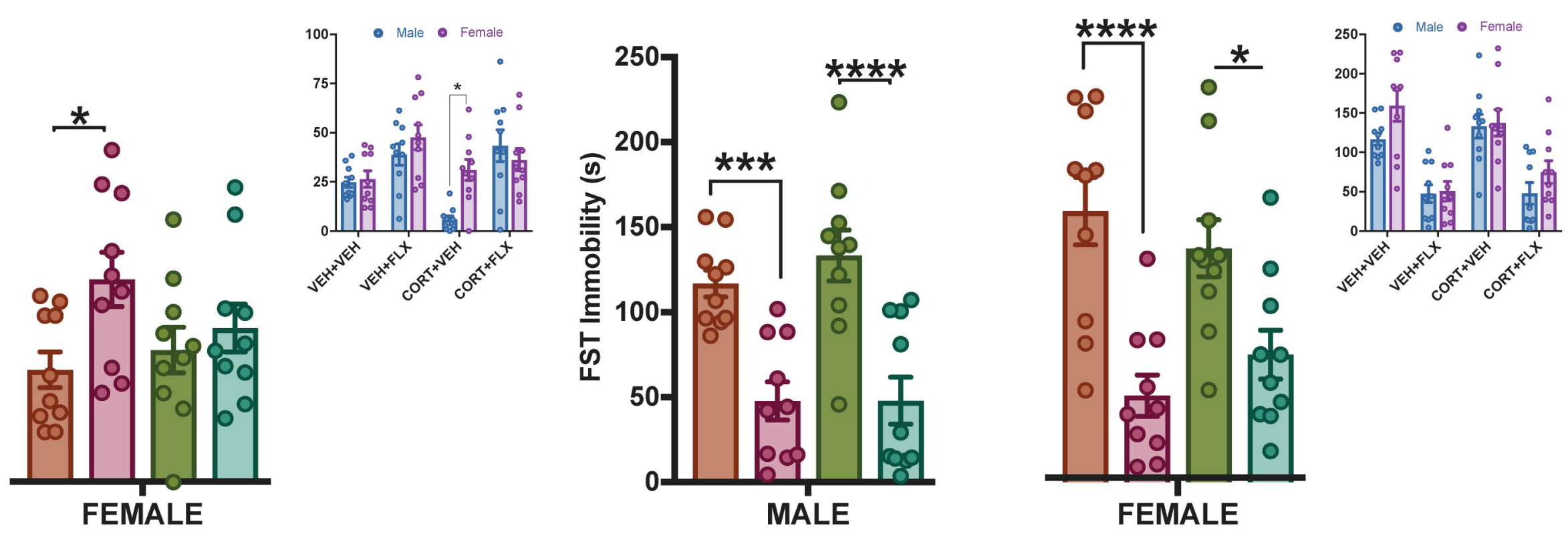

G

$\mathrm{H}$
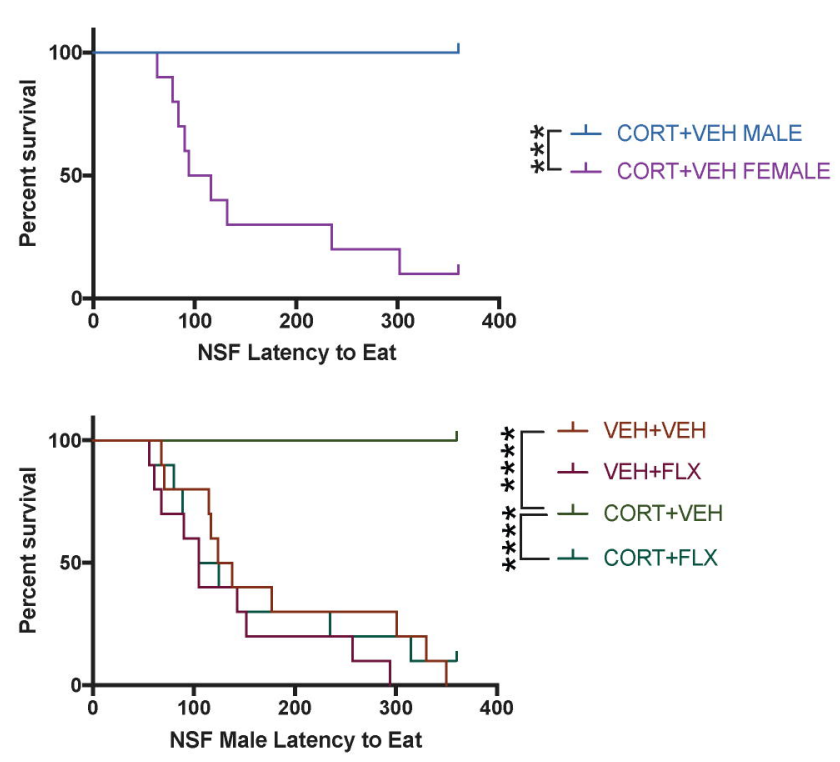


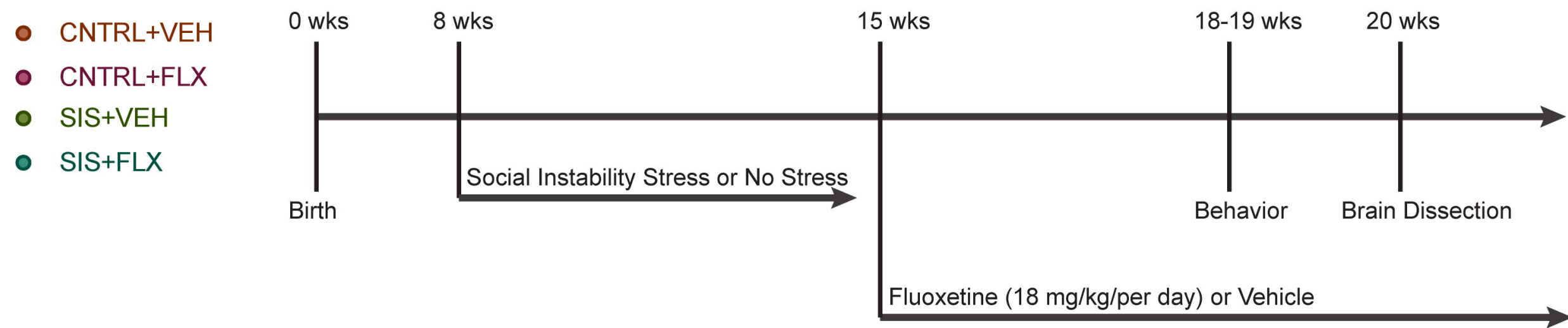

B

C

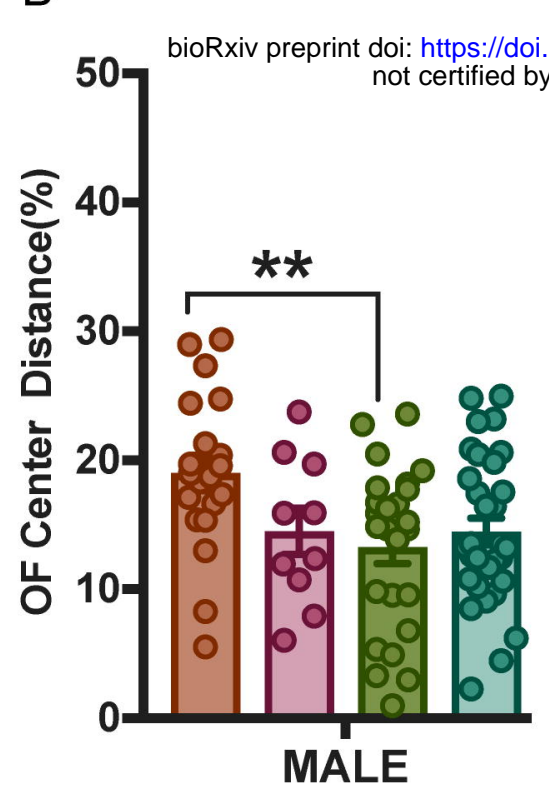

- Male 2 Female
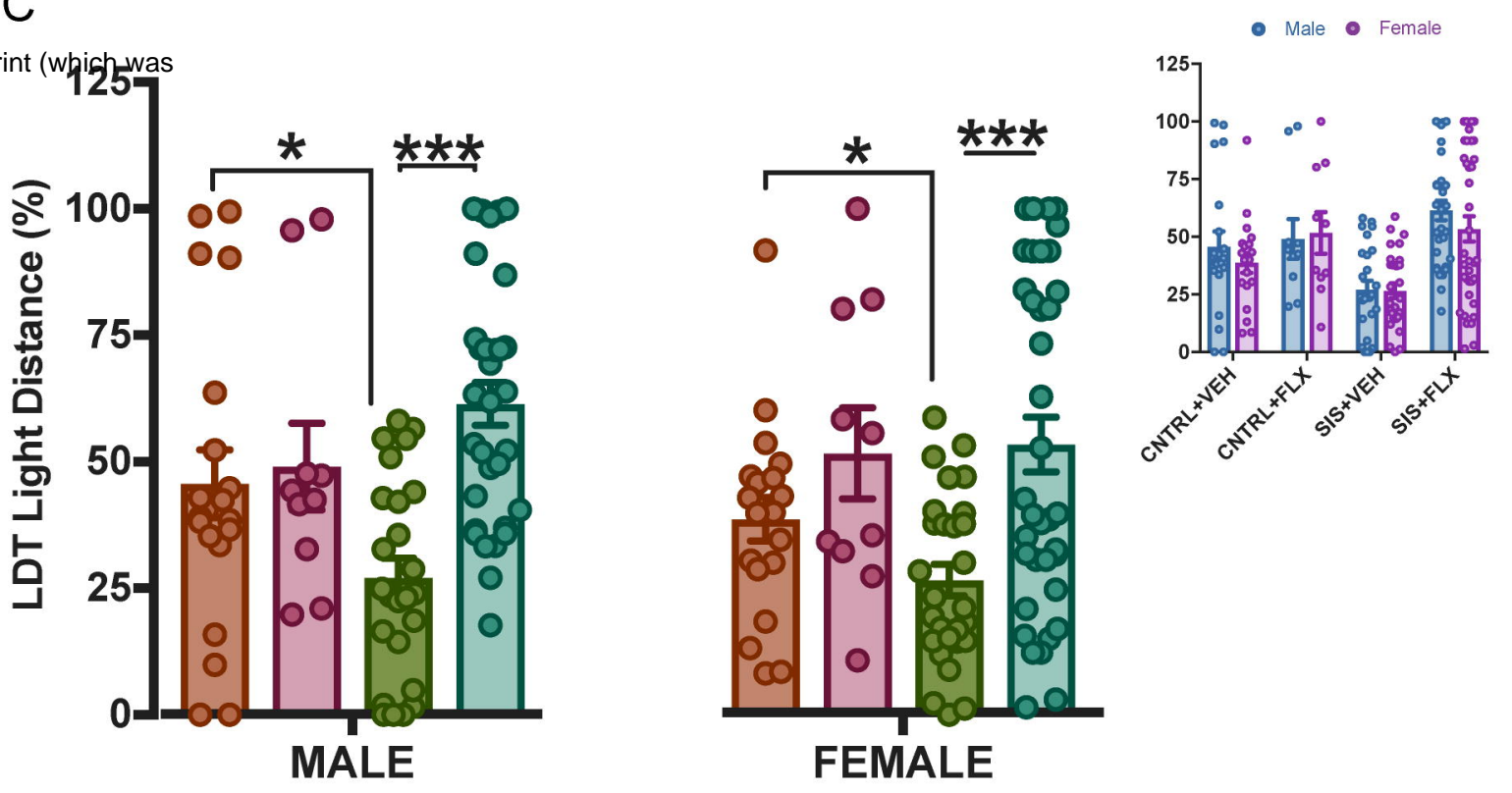

D
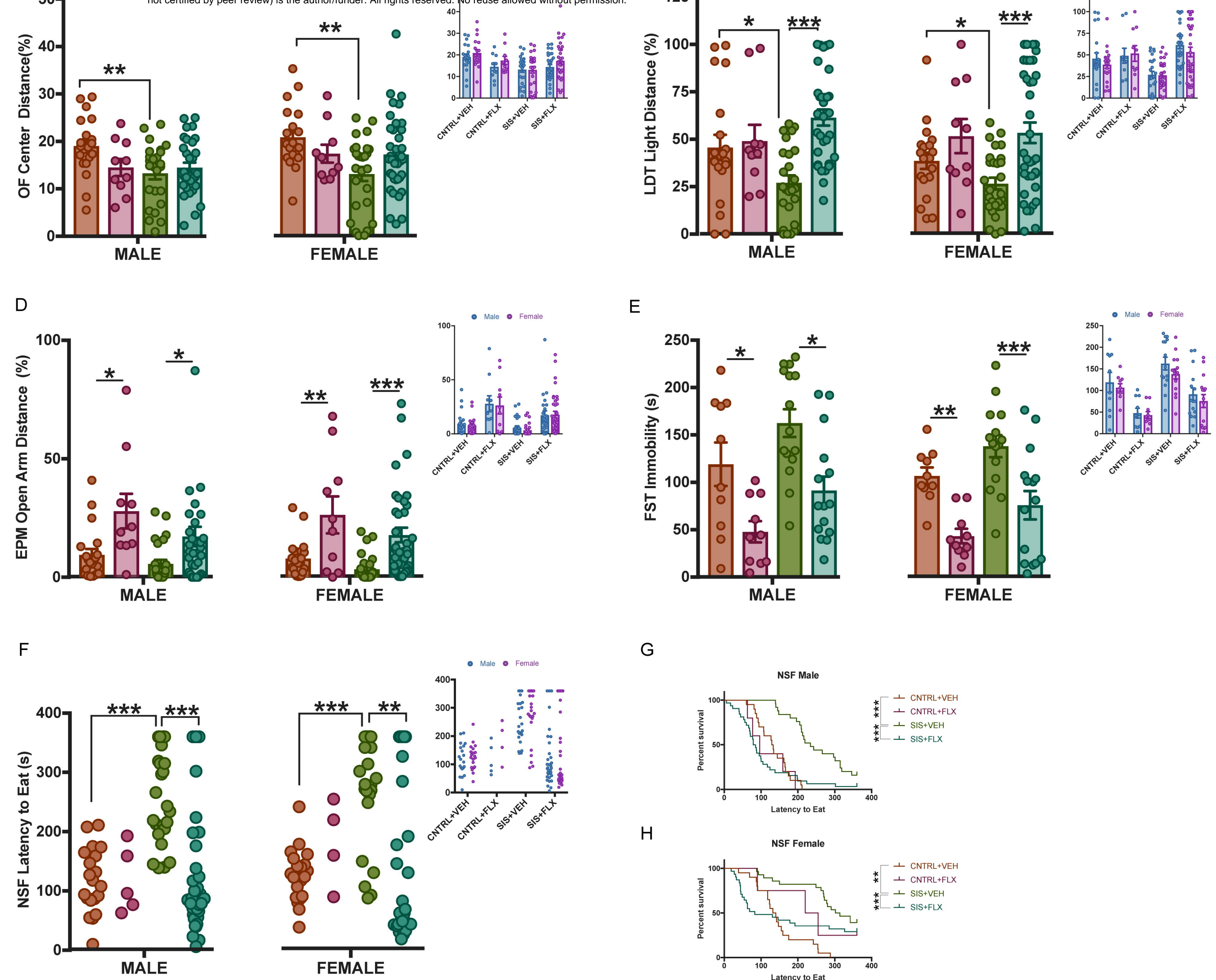

G

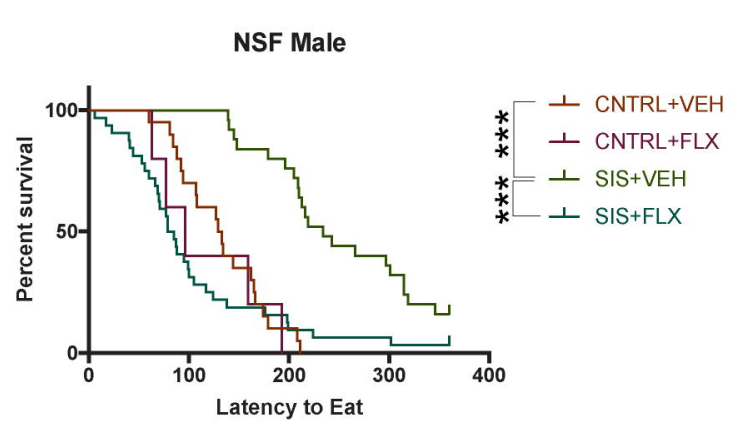

$\mathrm{H}$
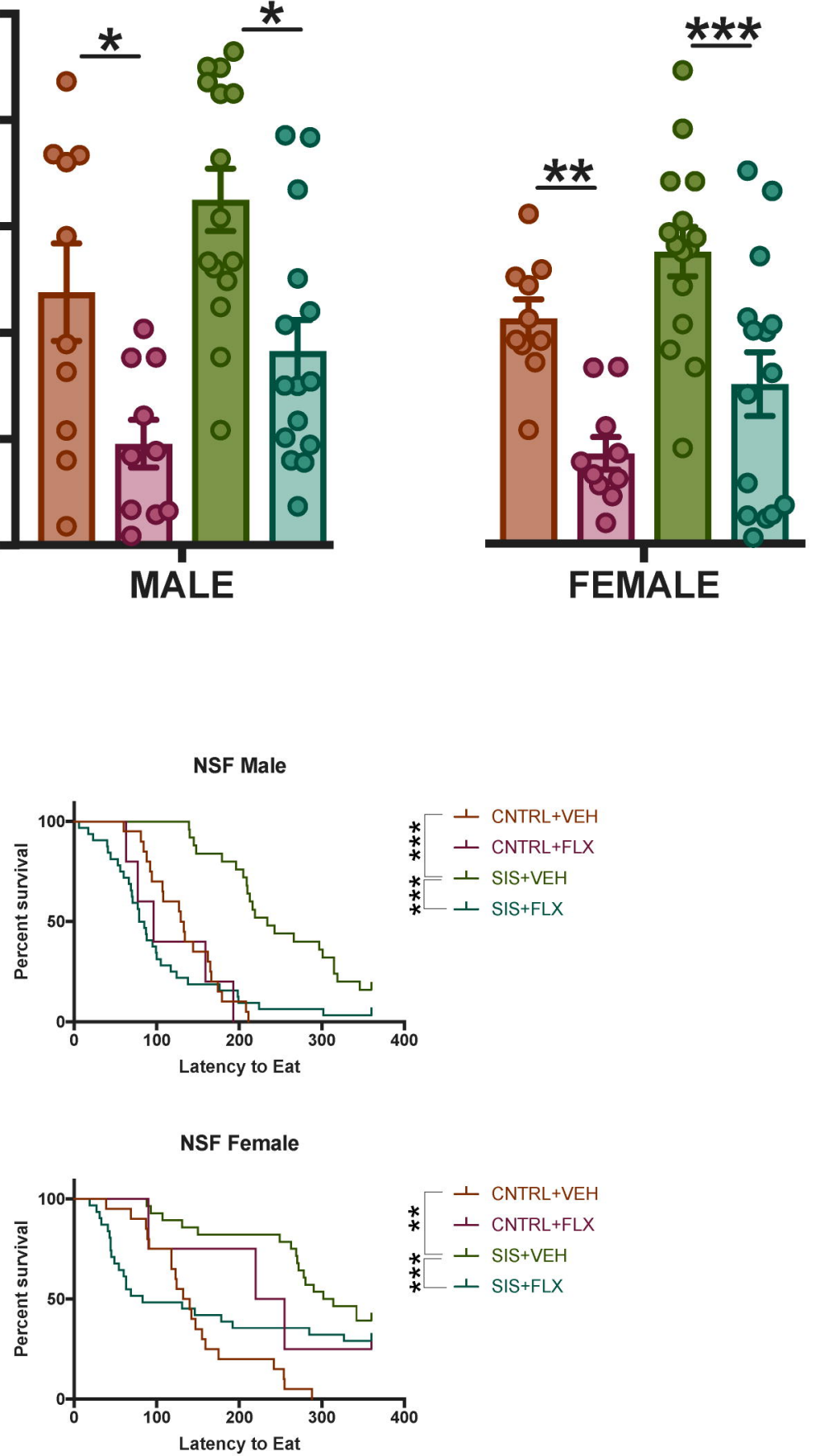

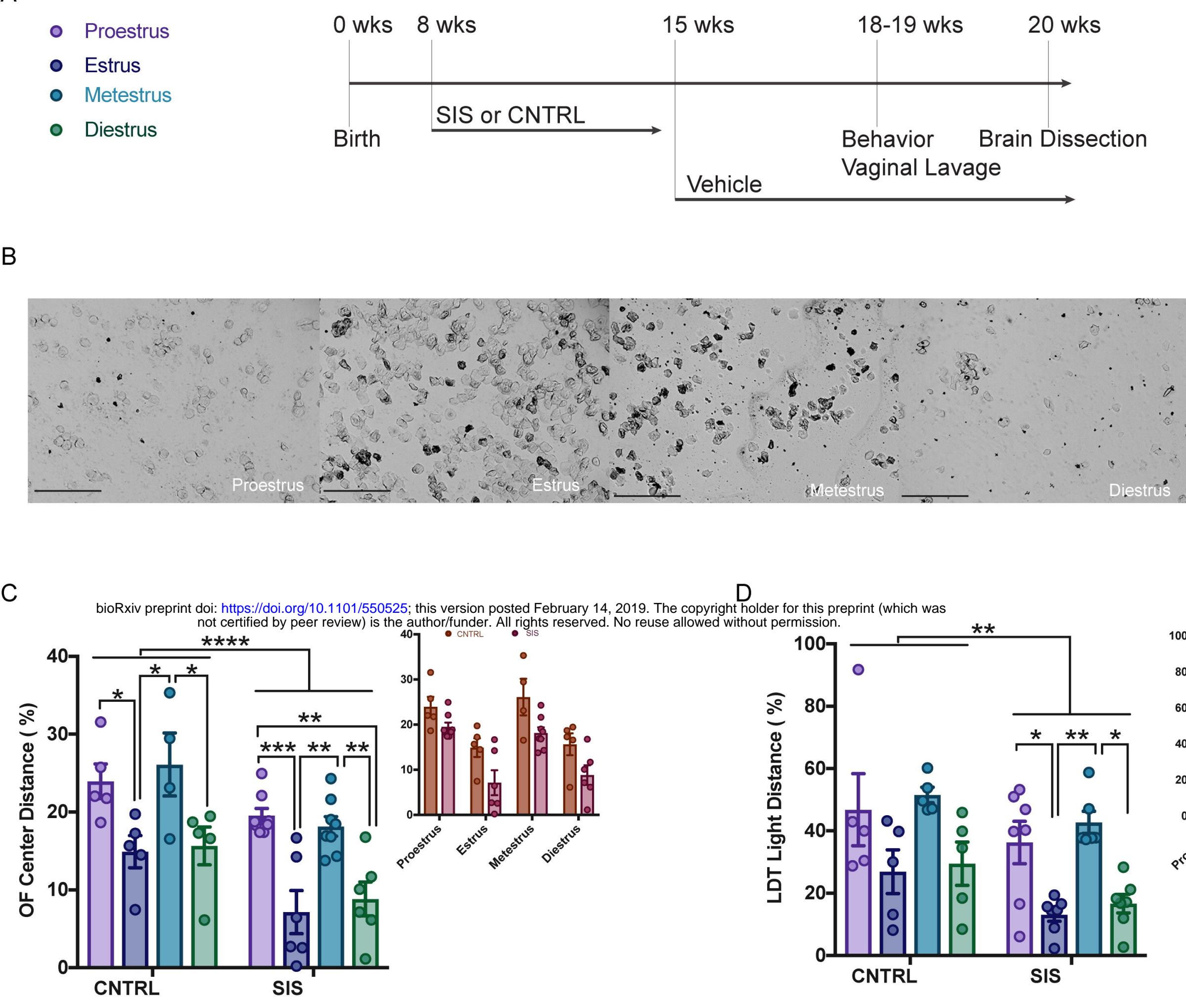

$\mathrm{D}$

der for this preprint (which was
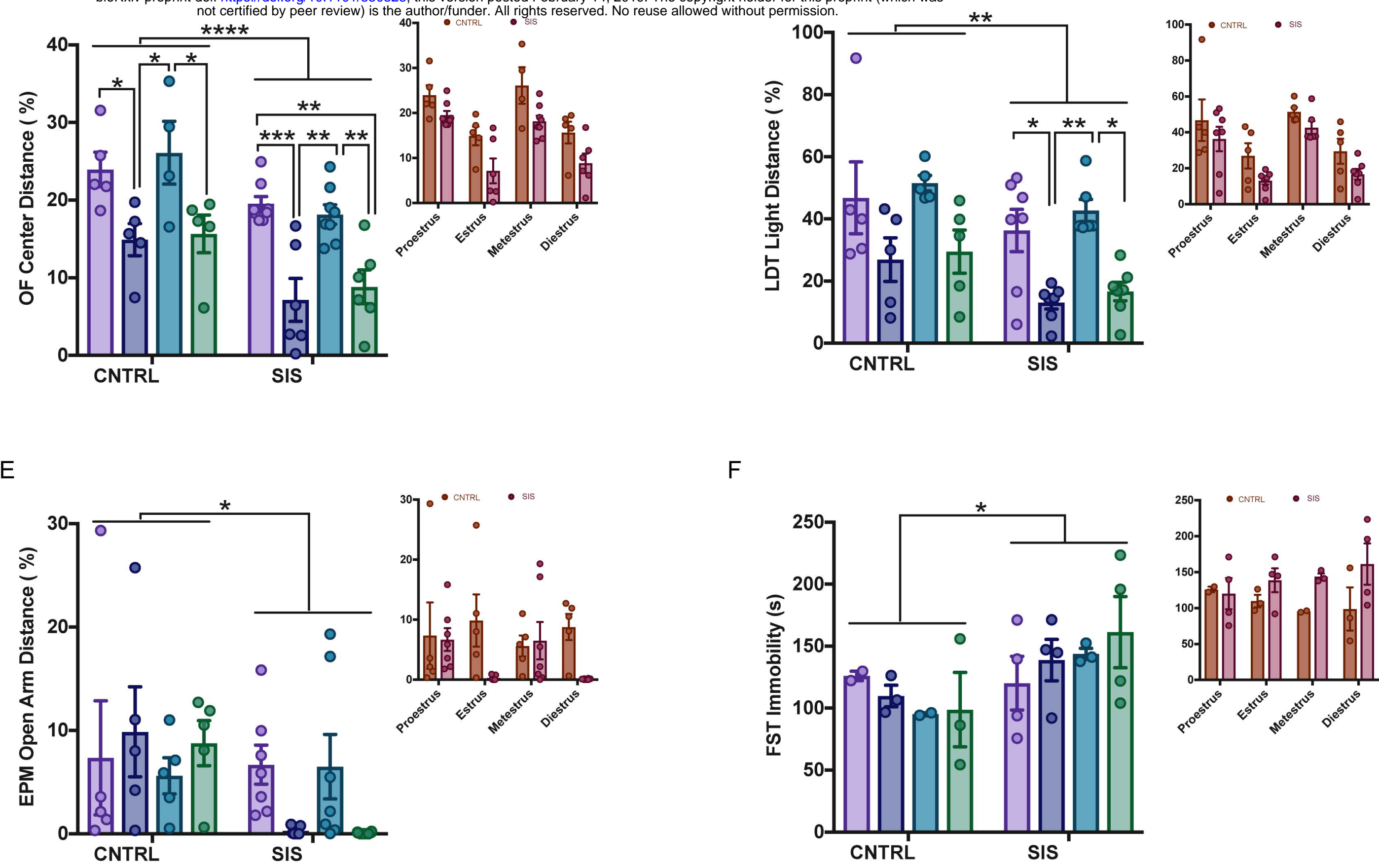

F

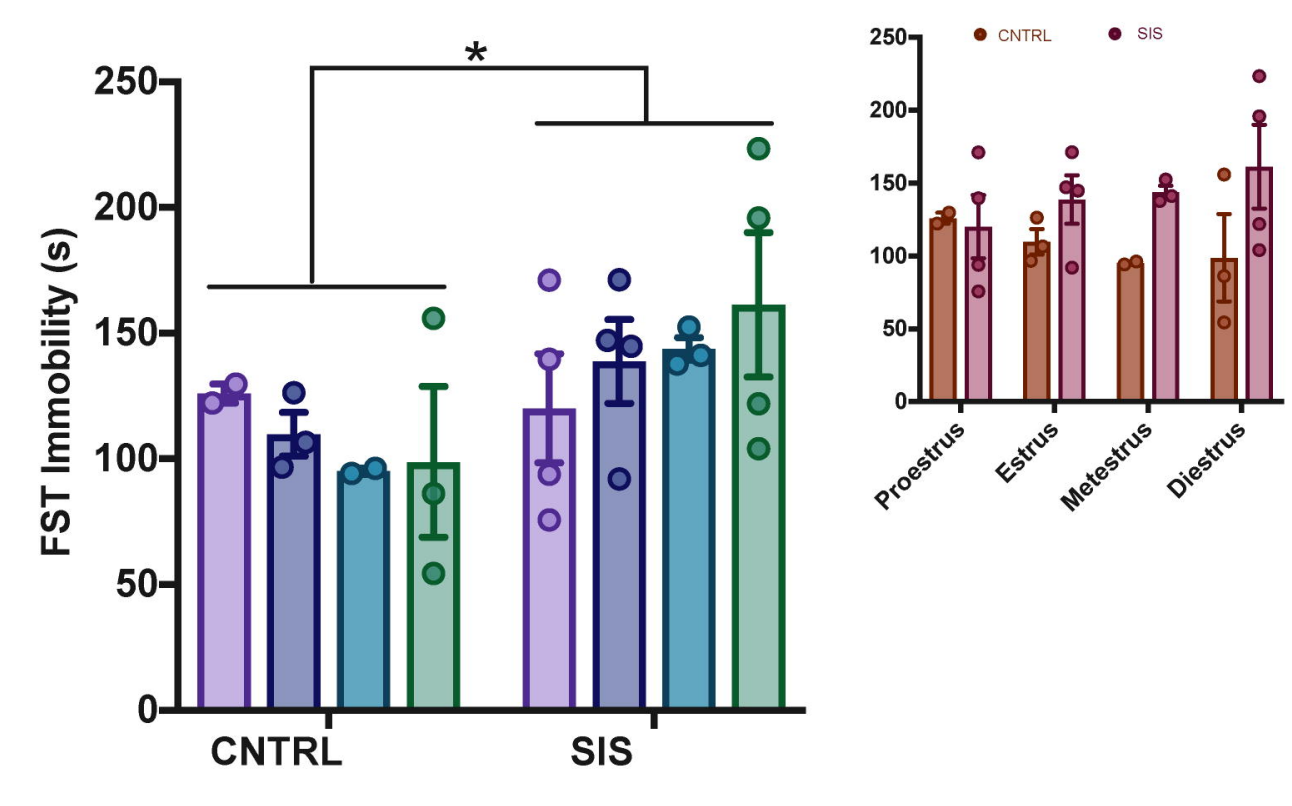

$\mathrm{H}$
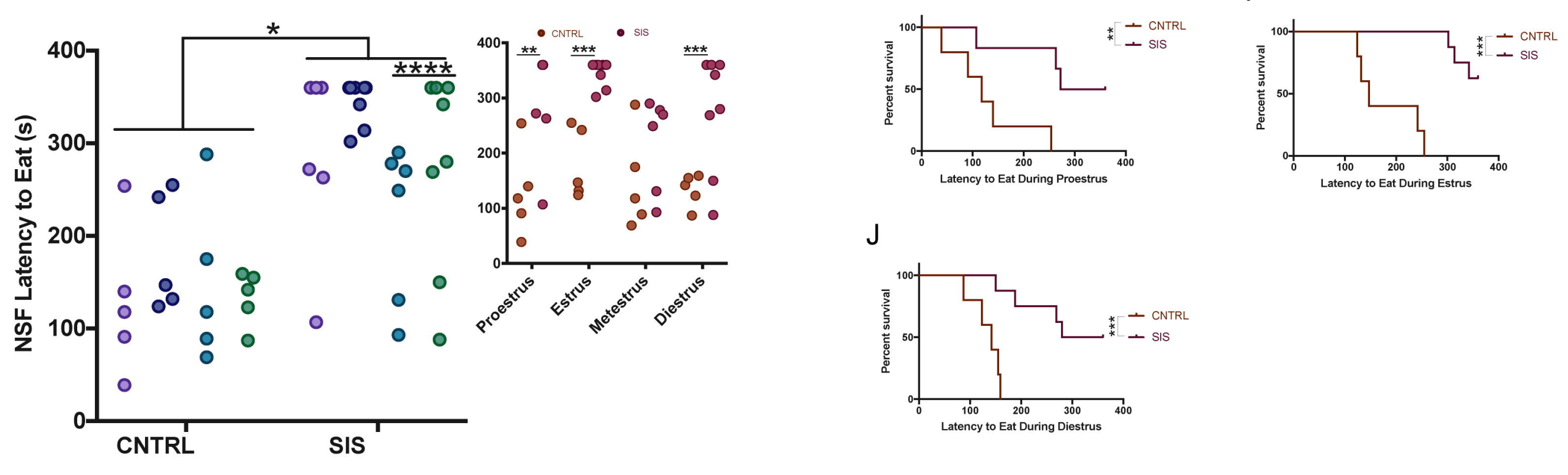

- Prior to SIS
O Cage Stress
Behavior

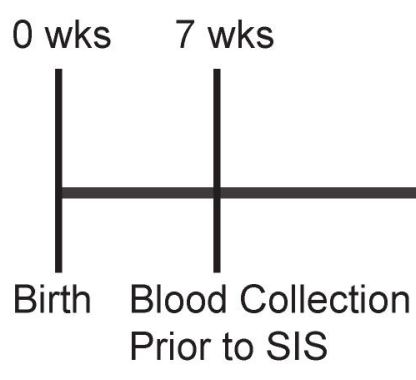

8 wks

s

14 wks

I

EPM Blood Collection

\section{0 wks}

SIS or CNTRL

C
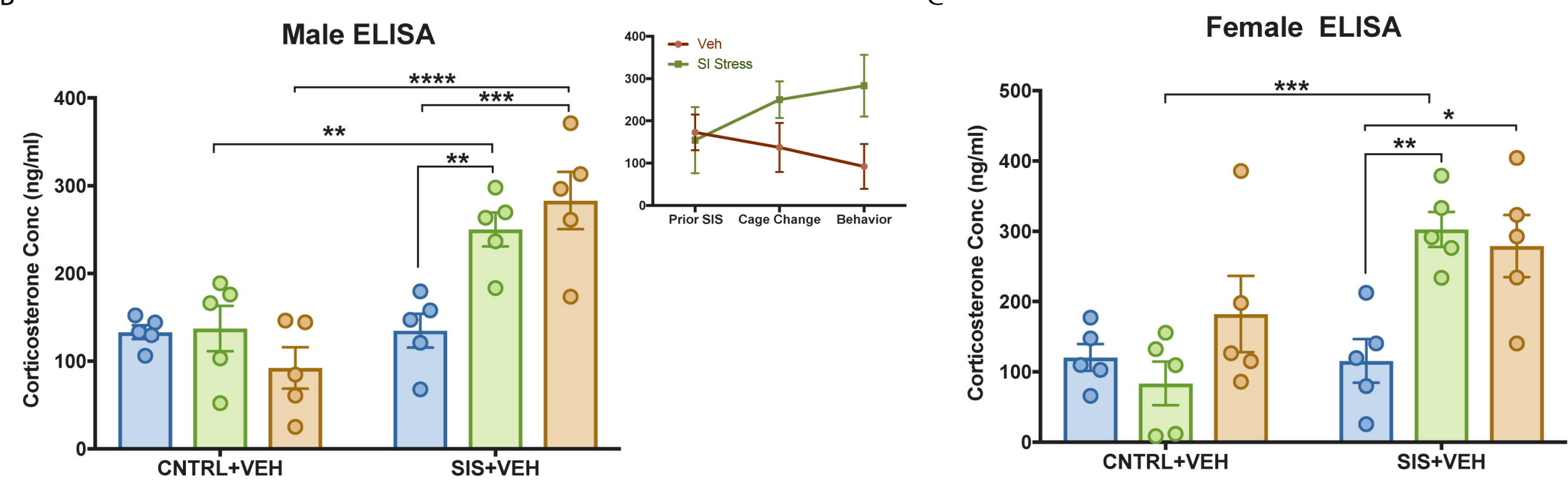
